\title{
Area laws in a many-body localized state and its implications for topological order
}

\author{
Bela Bauer ${ }^{1}$ and Chetan Nayak ${ }^{1,2}$ \\ ${ }^{1}$ Station Q, Microsoft Research, Santa Barbara, CA 93106-6105, USA \\ ${ }^{2}$ Physics Department, University of California, Santa Barbara, CA 93106, USA
}

\begin{abstract}
The question whether Anderson insulators can persist to finite-strength interactions - a scenario dubbed manybody localization - has recently received a great deal of interest. The origin of such a many-body localized phase has been described as localization in Fock space, a picture we examine numerically. We then formulate a precise sense in which a single energy eigenstate of a Hamiltonian can be adiabatically connected to a state of a non-interacting Anderson insulator. We call such a state a many-body localized state and define a manybody localized phase as one in which almost all states are many-body localized states. We explore the possible consequences of this; the most striking is an area law for the entanglement entropy of almost all excited states in a many-body localized phase. We present the results of numerical calculations for a one-dimensional system of spinless fermions. Our results are consistent with an area law and, by implication, many-body localization for almost all states and almost all regions for weak enough interactions and strong disorder. However, there are rare regions and rare states with much larger entanglement entropies. Furthermore, we study the implications that many-body localization may have for topological phases and self-correcting quantum memories. We find that there are scenarios in which many-body localization can help to stabilize topological order at non-zero energy density, and we propose potentially useful criteria to confirm these scenarios.
\end{abstract}

\section{INTRODUCTION}

Until relatively recently, it was generally assumed that all materials have non-vanishing electrical conductivity at nonzero temperature even in the presence of disorder. According to this conventional wisdom, an electron trapped in some potential energy well would always have some non-zero probability of being thermally-excited to a nearby site and, from there, to another, and so on. However, Basko et al. ${ }^{1,2}$ suggested, following ideas of Anderson ${ }^{3}$, that it could, instead, be possible for a system to remain an insulator even at non-zero temperature, an effect that they called many-body localization (MBL). According to their analysis, a weakly-interacting system of localized electrons cannot serve as its own heat bath (although phonons, which would necessarily be present in a solid but not in a system of ultra-cold atoms, could serve in that role). Consequently, the system cannot supply an electron with the energy that it needs to make a transition to a nearby site even when there is non-zero energy density, so that the system has far more total energy than is needed for this transition.

This hypothesis has profound consequences, not only for the electrical conduction of dirty metals and semiconductors (where, due to phonons, there will always be non-zero conduction at non-zero temperature, though it would be very small in a many-body localized state in the limit of weak electron-phonon interaction ${ }^{4}$ ), but also for the foundations of quantum statistical mechanics. The eigenstate thermalization hypothesis $(\mathrm{ETH})^{5,6}$ is an elegant justification of the microcanonical ensemble: expectation values of physical observables in individual energy eigenstates in generic (i.e. nonintegrable) systems are equal to their averages over all eigenstates of that energy - i.e. are equal to the predictions of the microcanonical ensemble. If, in a closed isolated system in an energy eigenstate, one focuses on any subsystem which is large but still much smaller than the whole system, the rest of the system can act as a heat bath, and the system will have the properties expected in thermal equilibrium. However, this must fail in systems in which many-body localization occurs. The rest of the system cannot act as a heat bath; rather than thermalize any added energy, it keeps it localized.

Basko et al. ${ }^{1,2}$ proposed many-body localization as an analogue of single-particle localization, in which a single particle moving on a lattice with (for instance) random on-site energies is unable, in an energy eigenstate, to explore the whole lattice and, instead, has an amplitude that decays exponentially with distance from some finite subset of the lattice. Similarly, if a many-body state has low energy density, which corresponds to low temperature, then it is unable to explore the entire Fock space of Slater determinants of single-particle eigenstates of the non-interacting disordered system and, instead, has appreciable amplitude only on some subspace. This picture of localization in Fock space has previously been discussed for quantum $\operatorname{dots}^{7-9}$.

Important progress was made by Oganesyan and Huse ${ }^{10}$, who noted that in a system with a maximum energy, such as a system of fermions on a lattice with a finite number of bands, it could be possible for many-body localization to occur even at "infinite temperature", meaning that it would hold for all energy eigenstates. They studied the energy level statistics of a system of interacting fermions in one dimension, and found that the energies, obtained by numerical diagonalization of the Hamiltonian, obeyed Poisson statistics for weak interactions and random-matrix level statistics for stronger interactions. The former corresponds to a many-body localized phase and the latter to a phase with extended states. However, the transition point between these two regimes drifted with system size, leaving open the possibility that all states are extended in the thermodynamic limit. The "infinite temperature" ensemble was further explored in Ref. 11.

Other probes of many-body localization, primarily involving the real-time dynamics of systems prepared in simple initial states, were investigated in a number of papers. Iyer $e t$ $a l .{ }^{12}$ computed the density auto-correlation function, configuration space participation ratio, and growth of the Renyi entanglement entropy in clean but quasiperiodic systems prepared 
in initial product states in the local occupation basis. They found two regimes, one in which these measures indicated that the subsequent evolution explored the entire Hilbert space, and another, interpreted as a many-body localized phase, in which it does not. Žnidarič et al. ${ }^{13}$, De Chiara et al. ${ }^{14}$, Bardarson et al. ${ }^{15}$ and Vosk and Altman ${ }^{16}$ studied the growth of the entanglement entropy in the evolution of the system from an initial product state in the local occupation basis. They found logarithmic growth, which was also interpreted as a possible indication of many-body localization. Theoretical explanations for this growth were recently discussed in Refs. 17 and 18. A logarithmic bound was also shown in Ref. 19 for the non-interacting case. (See Sec. V for further discussion of the growth of the entropy from an initial product state.)

In this paper, we put the Basko et al. ${ }^{1,2}$ picture to the test by numerical simulation of a system of spinless electrons with weak nearest-neighbor interactions in one dimension. In Section II, we show that, if we view the Slater determinants of (localized) single-particle wavefunctions as the nodes of a graph, then the matrix elements of the Hamiltonian only connect states that differ by local rearrangements of one or two electrons and are nearby in energy. Thus, motion on this graph occurs by "local" hops, roughly analogous to the motion of a single particle on a lattice. However, the coordination number of this graph scales linearly with the system size; in other words, lowest-order perturbation theory about the noninteracting state gives a contribution that is $O(N)$, where $N$ is the number of particles. In this respect, the many-body system seems different from the single-particle one, in which localization occurs in the strong disorder limit because perturbation theory in the hopping converges ${ }^{3,20}$. However, the failure of this extremely literal analogy between many-body localization and single-particle localization can be rather benign. Since, in a typical energy eigenstate, each particle can hop to a nearby state, there are $O(N)$ states with which a Slater determinant will have appreciable matrix element. Therefore, an energy eigenstate of an interacting system will generically not be a superposition of a finite number of eigenstates of the non-interacting system. However, the Hilbert spaces of manybody systems have dimension of $\mathcal{O}\left(e^{b N}\right)$, which is so large that, even if a state explores $\mathcal{O}\left(e^{a N}\right)$ states with $a<b$, this is still a very small fraction of the full Hilbert space.

In fact, we will argue in this paper that a system in a many-body localized phase explores only a relatively small and very specific subset of the full Hilbert space, namely the low-entanglement states. We will define many-body localization for an eigenstate of such a system as a state that may, in the following sense, be adiabatically connected to a state of the non-interacting system, which is a Slater determinant of localized single-particle states:

For a many-body localized energy eigenstate $|\psi\rangle$, there is a finite-depth local unitary transformation $U$ that will almost everywhere transform $|\psi\rangle$ into a Slater determinant of localized single-particle states, to within desired accuracy.

In other words, in a generic region of the lattice, the state looks similar to a product state. In Section III, we give a more precise version of this definition. Note that this is a definition that applies to a single eigenstate. The surprise is that there are excited states with a finite energy density above the ground state that satisfy this definition. We expect that the ground state of a disordered system in one or two dimensions (or, with sufficient disorder, a higher-dimensional system) will satisfy the definition given above (denoted Definition 2 in Section III A) even for strong interactions. However, for very weak interactions and strong disorder, the system may be in a regime in which almost all energy eigenstates satisfy this definition. We believe that this is closely related to the concept of "many-body localization at infinite temperature", discussed by Oganesyan and Huse ${ }^{10}$. We will say that such a system is in a many-body localized phase. It has been speculated that, for intermediate interactions, there is a critical energy density below which all states are many-body localized. However, we do not find evidence for a sharp energy density that is analogous to the mobility edge of single-particle localization in spatial dimensions $d>2$.

A possible consequence of Definition 2, which we discuss in more detail in Section III B, is that an energy eigenstate satisfying it will have a bipartite entanglement entropy with an 'area law': for almost all regions of size $L$ larger than the correlation length, the entanglement entropy $S$ between the region and the rest of the system has leading $L$-dependence

$$
S=\alpha L^{d-1}+\mathcal{O}\left(L^{d-2}\right)
$$

where $\alpha$ is a constant, independent of $L$, and $d$ is the spatial dimension. This is in sharp contrast to random quantum states, or highly excited (thermal) states of generic local Hamiltonians, which will obey a volume-law scaling ${ }^{21}$. For the ground state of a disordered, interacting system, such an area law was previously observed in Ref. 22. Here, however, we emphasize that in a many-body localized phase, an area law holds for nearly all eigenstates instead of merely for low-energy states. A further consequence is that the mutual information, which serves as an upper bound for all correlation functions ${ }^{23}$, decays exponentially. Note that entanglement properties have previously been used to identify phase transition $\mathrm{s}^{24}$, including transitions in disordered systems ${ }^{25}$.

In our definition of an MBL state and our statement of the area law, we used the terms "almost everywhere" and "almost all regions" because we find that there are rare regions in the system where the entanglement entropy is large. Furthermore, we wrote "nearly all eigenstates" in the previous paragraph because we find that there are states of arbitrarily high entanglement entropies at all energies, but the density of such states is exponentially-small. This is one reason why it is difficult to identify a sharp 'mobility edge' separating high and low entanglement entropy states.

We find clear evidence for two regimes in a system of interacting spinless fermions in 1D. In the weak-interaction regime, the median value of $S$ does not scale with $L$ (as expected from Eq. (1) with $d=1$ ). The density of states with high entanglement entropy falls off exponentially. Thus, our results are consistent with the existence of many-body localized states in this regime. In the strong-interaction regime, the median value of $S$ increases with $L$ in a manner consis- 
tent with a linear dependence on $L$ and the distribution of entanglement entropies is a Gaussian peaked near the median value. The situation is less clear for intermediate interaction strengths. It is possible that there is a sharp transition between the two regimes described above. An alternative possibility is that there is an intermediate phase in which there are comparable densities of high and low entropy states. We cannot, at present, rule out either of these possibilities.

One interesting possible consequence of many-body localization is that it may stabilize topological order in a manner analogous to the stabilization of zero-temperature topological qubits by single-particle localization. Here, we give a criterion for the stabilization of $2 \mathrm{D}$ topological order by manybody localization of quasiparticles. Our criterion relies on the use of Wilson loops. However, a related criterion in terms of entanglement entropy can also be formulated. This is potentially particularly useful when the topological phase is dual to the symmetry-unbroken phase of a magnetic system. Similar ideas were recently discussed in a paper by Huse et al. ${ }^{26}$.

The rest of this paper is structured as follows: In Section II, we discuss a 1D model of interacting spinless fermions in a random on-site potential from the perspective of localization in Fock space. In Section III, we introduce our definition of MBL in terms of continuation to a local product state with a finite-depth unitary transformation. We show how this implies an area law for the entanglement entropy and present calculations of the entanglement entropy for our 1D model. In Section IV we comment on some of the implications of these results for topological order and quantum memories at non-zero energy density. Finally, in Section V, we discuss our results.

\section{LOCALIZATION IN FOCK SPACE}

Basko et al. ${ }^{1,2}$ proposed that many-body localization could occur as an analogue of single-particle localization, in the sense that the state of the many-body system could be localized in Fock space. According to their analogy, the following correspondence holds:

$$
\begin{aligned}
& \text { Lattice Site } \leftrightarrow \text { Slater determinant } \Psi_{\alpha}^{\mathrm{Sl}} \\
& \text { of localized single-particle } \\
& \text { energy eigenstates } \\
& \text { Random On-Site Potential } \leftrightarrow \text { Hartree-Fock } \\
& \begin{array}{l}
\text { energy }\left\langle\Psi_{\alpha}^{\mathrm{Sl}}|H| \Psi_{\alpha}^{\mathrm{Sl}}\right\rangle \\
\text { of Slater determinant } \Psi_{\alpha}^{\mathrm{Sl}}
\end{array} \\
& \text { Hopping } \leftrightarrow \text { Interaction }
\end{aligned}
$$

In this section, we briefly discuss this analogy, while we postpone a discussion of our numerical results to App. C. For another recent numerical study of this analogy, see Ref. 27.

For the sake of concreteness, let us consider the following
Hamiltonian in one dimension:

$$
\begin{aligned}
H & =H_{0}+H_{\mathrm{int}} \\
H_{0} & =-t \sum_{i=1}^{L-1}\left(c_{i}^{\dagger} c_{i+1}+c_{i+1}^{\dagger} c_{i}\right)+\sum_{i=1}^{L} w_{i} n_{i} \\
H_{\mathrm{int}} & =V \sum_{i=1}^{L-1} n_{i} n_{i+1},
\end{aligned}
$$

where $c_{i}^{\dagger}$ creates a spinless fermion on site $i$, and $n_{i}=c_{i}^{\dagger} c_{i}$. The $w_{i}$ are uniformly chosen from $w_{i} \in[-W, W]$. We use open boundary conditions. The non-interacting problem $H_{0}$ is readily solved to obtain the eigenvalues $\varepsilon_{n}$ and eigenvectors $\phi_{n}(i)$. We can then rewrite the problem as follows:

$$
H=\sum \varepsilon_{n} d_{n}^{\dagger} d_{n}+\sum_{i j k l} V_{i j k l} d_{i}^{\dagger} d_{j}^{\dagger} d_{k} d_{l},
$$

where $d_{n}^{\dagger}$ creates a fermion in the single-particle state $\phi_{n}(i)$. Note that in this formalism, the single-particle states $\phi_{n}(i)$ are obtained as solutions purely of the non-interacting problem and not as solutions of a Hartree-Fock equation for the interacting Hamiltonian. Finally, this can be recast in the following form:

$$
H=\sum_{\vec{\alpha}} \mu_{\alpha}|\vec{\alpha}\rangle\left\langle\vec{\alpha}\left|+\sum_{\vec{\alpha} \neq \vec{\beta}} V_{\beta \alpha}\right| \vec{\beta}\right\rangle\langle\vec{\alpha}| .
$$

In this model, the on-site potential $\mu_{\alpha}$ is given by the HartreeFock energy of the single-particle Slater determinants, which consists of the non-interacting part of the original Hamiltonian as well as the diagonal part of the interaction term. The hopping term $V_{\beta \alpha}$ stems purely from the interaction term. Full definitions for all these models are given in App. C.

It was argued by Basko et al. ${ }^{1,2}$ that Hamiltonian (3) has a very particular structure, namely that due to the localization of the single-particle orbitals $\phi_{n}(i)$, the matrix elements $V_{i j k l}$ fall off exponentially with separation between the states $\phi_{i}$ and $\phi_{k}$ or $\phi_{l}$ and between $\phi_{j}$ and $\phi_{k}$ or $\phi_{l}$. They further argued that these matrix elements decay very quickly with the difference between single-particle energies. This holds for a quantum dot in the diffusive regime, where quantum interference corrections can be neglected ${ }^{28,29}$, whereas we will be dealing with the strong disorder regime in this paper. We therefore confirm this condition numerically in App. C.

In order to be able to apply a single-particle localization perspective to the Fock space hopping problem (4), it is necessary to understand the properties of the (random) graph on which this hopping Hamiltonian is defined. To make progress on this, we calculate an effective coordination number

$$
z=\left\langle z_{\alpha}\right\rangle, \quad z_{\alpha}=\sum_{\beta \neq \alpha} \frac{V_{\beta \alpha}}{\left|\mu_{\beta}-\mu_{\alpha}\right|}
$$

where $\langle\cdot\rangle$ indicates averaging over $\alpha$ as well as disorder realizations. As discussed in more detail in App. C, we observe that the effective coordination number scales linearly in the system size, $z \sim L$, independently of the parameters of our 
model, i.e. in both the regime where we expect a delocalized phase and the regime in which we expect an MBL phase. This divergence is easily understood by the following argument: in a generic Slater determinant $|\vec{\alpha}\rangle$ at half-filling, there are $N=L / 2$ fermions that can hop within a small part of the system, whose volume is controlled by the localization length and essentially is $\xi_{\text {loc }}^{d}$. In our Fock space model, the number of Slater determinants $|\vec{\beta}\rangle$ to which the system can "hop" is therefore also proportional to the number of fermions $N=L / 2$, and hence our coordination number $z \sim N$. In App. C, we confirm this argument by calculating the dependence of $z$ on the number of fermions, $N$, in a system of fixed size $L$.

We conclude that the effective coordination number scales as $N$. This is not very deep: each of the $N$ electrons can make a transition to a nearby (in both location and energy) state. However, this means that MBL is not likely to be a simple analogue of single-particle localization, with Eq. (4) playing the role of $H_{0}$ in Eq. (2) for a single particle. This is further illustrated by consideration of the inverse participation ratios:

$$
I_{\psi}=\sum_{\alpha}|\langle\alpha \mid \psi\rangle|^{4}
$$

Precisely at $V=0$, the inverse participation ratio is 1 in every eigenstate. As $V$ is increased, we find that the inverse participation ratios decrease linearly with $V$. But our calculated values of $I_{\psi}$ as a function of $V$ do not show simple scaling behavior as a function of system size. Moreover, we expect that $I_{\psi} \sim e^{-a N}$ even in an MBL phase because each electron can hop to $O(1)$ nearby localized states, so it would be difficult to use $I_{\psi}$ to distinguish an MBL phase from a metallic state.

\section{ADIABATIC CONTINUITY AND ENTANGLEMENT ENTROPY}

\section{A. Definition of a Many-Body Localized State}

In this paper, we adopt the point of view that when all of the states of a system are many-body localized, they should be adiabatically connected to the localized states of the corresponding non-interacting system. However, it is difficult to make this notion precise by considering direct analogues of criteria used in single-particle localization, such as inverse participation ratios, since even weak interactions will mix exponentially many single-particle Slater determinants to obtain the eigenstate of the interacting system.

For this reason, the definition of many-body localization that we propose in this paper is, as stated in the introduction:

For a many-body localized energy eigenstate $|\psi\rangle$, there is a finite-depth local unitary transformation $U$ that will almost everywhere transform $|\psi\rangle$ into a Slater determinant of localized single-particle states, to within desired accuracy.

In this section, we will try to make the words "almost" and "to within desired accuracy" more precise. For the most part, we will assume (without justification) in this paper that the latter caveat does not matter, but the first one will play a role in our calculations. The first step in making our definition more precise is to define the "localization depth" of a state (for a definition of a local unitary transformation of depth $D$ and localized single-particle states, see Appendix A):

Definition 1. A state $|\psi\rangle$ on a lattice $\mathcal{L}$ has localization depth $D$ to accuracy $(\epsilon, k)$ in some (not necessarily connected) subset $\mathcal{A}$ if there exists a local unitary transformation $U$ of depth $D$ and a Slater determinant of localized single-particle states $\left|\Psi^{\mathrm{Sl}}\right\rangle$, such that the reduced density matrices,

$$
\rho_{\mathcal{B}}=\operatorname{Tr}_{\mathcal{L} \backslash \mathcal{B}}|\psi\rangle\left\langle\psi\left|, \quad \rho_{\mathcal{B}}^{\mathrm{Sl}}=\operatorname{Tr}_{\mathcal{L} \backslash \mathcal{B}}\right| \Psi^{\mathrm{Sl}}\right\rangle\left\langle\Psi^{\mathrm{Sl}}\right|,
$$

satisfy the property that

$$
\operatorname{Tr}\left(\left|\rho_{\mathcal{B}}-U \rho_{\mathcal{B}}^{\mathrm{Sl}} U^{\dagger}\right|\right)<\epsilon .
$$

for all connected $\mathcal{B} \subset \mathcal{A}$ with $\operatorname{vol}(\mathcal{B})=k$.

Here, $\operatorname{Tr}(|\cdot|)$ denotes the trace norm distance, which for reduced density matrices of two states has the operational meaning that it quantifies how well the two states can be distinguished by measurements only on these reduced density matrices $^{30-32}$. This definition thus formalizes the notion that such a state shares all $k$-local properties of a localized Slater determinant, i.e. properties that can be measured using $k$-local operators. A key requirement is that a single transformation $U$ and a single Slater determinant $\left|\Psi^{S l}\right\rangle$ are used for all $\mathcal{B}$; if one allows a different $U$ or $\left|\Psi^{\mathrm{Sl}}\right\rangle$ for each $\mathcal{B}$, the above definition can always be fulfilled with $D \sim k$. In our definition, we require that $\left|\Psi^{\mathrm{Sl}}\right\rangle$ be a Slater determinant of localized singleparticle states, as defined in Appendix A, rather than the basis of site occupation numbers so that our unitary $U$ does not have to remove the exponential tails of localized states, as we discuss further in the next subsection.

Of course the localization depth $D$ will generally grow with $k$, i.e. as more long-ranged properties of the system are being captured. A similar picture emerges when describing gapped states in one dimension as local unitary circuits applied to a product state, which is equivalent to describing them with a matrix-product state of bond dimension $M \sim \exp (D)$ : while local properties may be accurately described by a circuit of finite depth $D$ even as $L \rightarrow \infty^{33}$, the bond dimension $M$ will have to grow polynomially with $N$ if some fixed accuracy is demanded for the density matrix on $N$ sites $^{34,35}$. In our case, the localization depth $D$ would depend on $\operatorname{vol}(\mathcal{A})$ if one were to demand $\mathcal{B}=\mathcal{A}$; in order to avoid this dependence and to facilitate approaching the thermodynamic limit below, we only require $k$-local properties to match to a localized Slater determinant, allowing us to obtain a finite $D$ independent of $\operatorname{vol}(\mathcal{A})$, for a given $k$.

Of course, the above definition can always be fulfilled if $D$ is allowed to scale sufficiently $\operatorname{rapidly}$ with $\operatorname{vol}(\mathcal{A})-$ in other words, in a finite-size system, every state has a finite localization depth in all subsets. The distinction between many-body localized states and extended ones must, therefore, be the scaling of the localization depth with the system size. We now propose a definition of a many-body localized state that draws 
a line between the two types of states. For simplicity, we give a definition on the hypercubic lattice $\mathbb{Z}^{d}$, but this definition can be generalized to an arbitrary lattice or even an arbitrary triangulation.

Definition 2. We will say that an energy eigenstate $|\psi\rangle$ of a Hamiltonian $H$ on $\mathbb{Z}^{d}$ is an MBL state if, for any $\epsilon>0$ and any $0<f<1$, there exists a sequence of hypercubic regions $C_{i} \subset \mathbb{Z}^{d}$ satisfying $C_{1} \subsetneq C_{2} \subsetneq \ldots$ and a sequence of subsets $\mathcal{A}_{i} \subset C_{i}$ satisfying $\mathcal{A}_{1} \subsetneq \mathcal{A}_{2} \subsetneq \ldots$ and $\operatorname{vol}\left(\mathcal{A}_{i}\right) / \operatorname{vol}\left(C_{i}\right)>$ $f$ such that the following holds: $D \equiv \lim _{i \rightarrow \infty} D_{i}$ is finite for any finite $k$, where $D_{i}$ is the localization depth of $|\psi\rangle$ to accuracy $(\epsilon, k)$ on $\mathcal{A}_{i}$.

In other words, an MBL state can be approximately transformed into a Slater determinant of localized single-particle orbitals almost everywhere by a local unitary transformation of finite depth. We emphasize that, at this point, our definition applies to a single eigenstate of a Hamiltonian. The details of the Hamiltonian determine whether any of its eigenstates are MBL states. However, these details - e.g. whether there is a random potential, whether the interactions are short-ranged or long-ranged, etc. - do not enter our definition of an MBL state. To the best of our knowledge, such a definition has not been previously formulated. (However, see Refs. 18 and 36 where similar ideas are discussed.) Most previous discussions have focused on the dynamical properties of Hamiltonians. Our treatment can be more directly compared to those discussions when, in Section III D, we give a definition of a manybody localized phase that applies directly to the properties of a Hamiltonian.

This definition provides an attempt to formalize the basic idea that states of a many-body localized system are adiabatically connected to states of a non-interacting Anderson insulator. A non-interacting Anderson insulator has the unusual property that all of its states below the mobility edge are Slater determinants of localized single-particle orbitals. While this is also true for the ground state of a band insulator (since fully occupied bands can be expanded in localized Wannier orbitals), it is not true for its excited states at non-zero energy density. It is also not true for arbitrary eigenstates (including the ground state) of symmetry-protected topological phases $^{37,38}$ or gapless phases. Our definition essentially boils down, then, to the idea that this unusual property of a noninteracting Anderson insulator can also hold, to within desired accuracy, in an interacting system. Note that our definition is not precisely the same as a failure of the ETH hypothesis ${ }^{5,6}$; one can imagine non-ergodic states that aren't MBL states ${ }^{39}$.

In this respect, we can connect Definition 2 to a definition given by Basko et al. ${ }^{1,2}$ in their pioneering work. Although our definition applies to an individual energy eigenstate rather than a range of temperatures and focusses on the relation to a Slater determinant via local unitary transformations of finitedepth, it has similar consequences if it holds for almost all states of a system. Consider the following statement of manybody localization given by Basko et al. ${ }^{1,2}$ : Label the energy eigenstates of the system $|k\rangle$ and consider matrix elements of a bounded local operator $X$,

$$
C_{k^{\prime} k} \equiv\left\langle k^{\prime}|X| k\right\rangle
$$

(Basko et al. ${ }^{1,2}$ consider the operator $c_{\alpha}^{\dagger} c_{\beta}$, but their statement can be generalized to any local operator $X$.) Then, one could define MBL by requiring that if all $|k\rangle,\left|k^{\prime}\right\rangle$ are MBL states in subset $\mathcal{A}$, then

$$
\frac{\sum_{k^{\prime}}\left|C_{k^{\prime} k}\right|^{4}}{\left(\sum_{k^{\prime}}\left|C_{k^{\prime} k}\right|^{2}\right)^{2}}
$$

has a non-zero limit in thermodynamic limit, while in the metallic phase, it has a vanishing limit. The sum in Eq. 8 will have a non-zero limit if there is one term in the sum that does not scale with system size. This property can be inherited from the non-interacting case as follows. We can insert the local unitary transformation $U_{k}$ for each state $|k\rangle$ to obtain

$$
C_{k^{\prime} k}=\left\langle k^{\prime}\left|U_{k^{\prime}}^{\dagger} U_{k^{\prime}} X U_{k}^{\dagger} U_{k}\right| k\right\rangle=\left\langle\Psi_{k^{\prime}}^{\mathrm{Sl}}\left|U_{k^{\prime}} X U_{k}^{\dagger}\right| \Psi_{k}^{\mathrm{Sl}}\right\rangle
$$

If $X$ has support only on $\mathcal{A}$ and $\mathcal{A}^{\prime}$, the regions of the two states where $U_{k}, U_{k^{\prime}}$ have finite-depth, then $U_{k^{\prime}} X U_{k}^{\dagger}$ is also a local operator and $\left|\Psi_{k}^{S l}\right\rangle$ is a Slater determinant of localized single-particle wavefunctions.

\section{B. Area-Law for the Entanglement Entropy}

A key proposition of this paper is that many-body localized states at non-zero energy, which meet the requirements of Definition 2, have an area law for the entanglement entropy. This area law will lead to a more practically useful definition for an MBL state since it is generically difficult to find the finite-depth local unitary transformation $U$ required by Definition 2, whereas entanglement properties are readily calculated by a variety of computational and analytical approaches.

In Section II, we have encountered the issue that even a localized system may explore an exponentially large part of its Hilbert space. The same issue is also encountered when attempting to accurately describe the low-energy states of a gapped system such as a band insulator. Expanding a lowenergy state of such a system in a local basis, one finds contributions from exponentially-many local product states. At the same time, it is known that the ground states of band insulators - in fact, the ground states of most local Hamiltonians only have overlap with a tiny subset of the full Hilbert space, namely that of low-entanglement states, which obey an area law ${ }^{21,40-42}$ or, in the case of many gapless systems, an area law with a multiplicative logarithmic correction. In one dimension, the area law for the ground state of systems with a finite correlation length has been firmly established ${ }^{35}$, while a logarithmic correction is expected for the ground state of critical (scale-invariant) systems ${ }^{24,43,44}$, including some random ones $^{45}$. In higher dimensions, the situation is more complicated and rigorous results are only available for the ground states of free systems ${ }^{46-48}$ and certain classes of interacting systems. In all of these systems, a volume law scaling will generically be observed for high-energy eigenstates. Here, we propose that many-body localized states have the highly unusual property that they are excited states satisfying an area law. As we now discuss, it is reasonable to conjecture that 


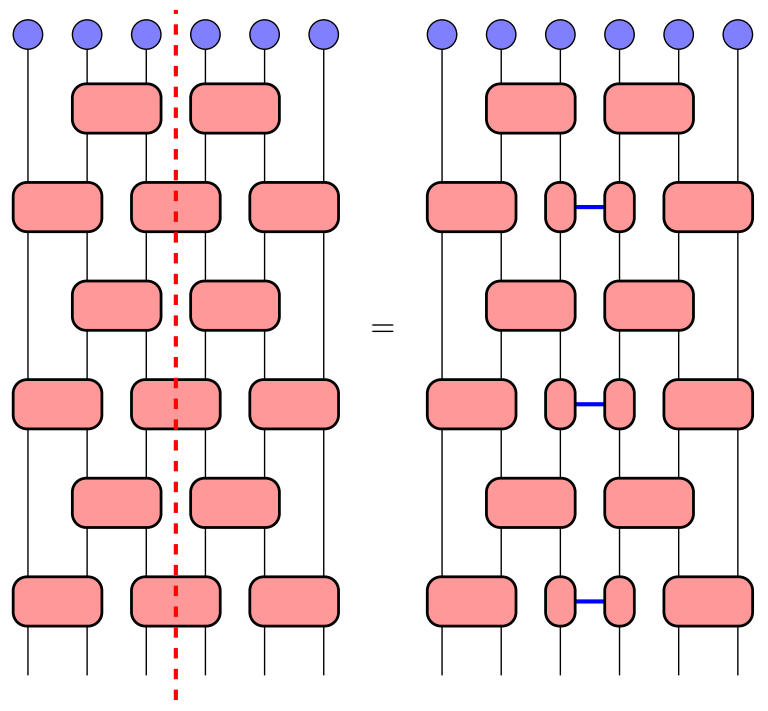

FIG. 1. Transformation of a product state $|\phi\rangle$ (blue dots, top) into an entangled state by a finite-depth 2-local (possibly unitary) transformation of depth $D=3$ (see Def. 4 in App. A). The amount of entanglement in the state after transformation is controlled by the number of operators that extend across a cut (such as indicated by the dashed red line on the left), i.e. by the depth of the circuit $D$. Decomposing the operators using an SVD leads to the picture on the right, which is similar to a Schmidt decomposition. The maximum entanglement is proportional to $D$.

the area law follows from our definition of an MBL state and from properties of non-interacting Anderson insulators.

Consider, first, the origin of such an area law for a system of non-interacting spinless fermions, where we fill some set of single-particle orbitals $\phi_{n}(i)$. Any eigenstate of such a system can be written in the form

$$
|\psi\rangle=\left(\sum_{i} \phi_{1}(i) c_{i}^{\dagger}\right)^{n_{1}}\left(\sum_{i} \phi_{2}(i) c_{i}^{\dagger}\right)^{n_{2}} \ldots|0\rangle,
$$

where $n_{i}=0,1$ are the occupation numbers of the singleparticle orbitals. Let us assume that the single-particle orbitals $\phi_{n}$ are strictly local, i.e. they have support only on $m$ contiguous sites. We can then pictorially represent the state as a product of local operators acting on a product state, as shown in the left panel of Fig. 1. Note that these operators are not unitary, but this does not matter for the purpose of our arguments here. Using this construction, we have successfully decomposed the state $|\psi\rangle$ into a tensor network. We can now use standard tensor network arguments to read off the maximum amount of bipartite entanglement contained in the state $|\psi\rangle$ : Consider splitting every operator crossing the dashed red line indicated in Fig. 1 using a singular value decomposition to obtain the picture shown in the right panel. The number of non-vanishing singular values for each operator is bounded by $d^{m}$, where $d$ is the dimension of the local Hilbert space (e.g., $d=2$ for spinless fermions). The rank of a reduced density matrix describing the left or right part is then bounded by this number to the power of the number of operators cut, i.e. the number of blue lines in the right panel, i.e. it is bounded by
$\left(d^{m}\right)^{D}$, where $D$ is the depth of the circuit. It follows that the bipartite entanglement entropy is bounded by

$$
S \leq m D \log d
$$

The minimal depth $D$ of the tensor network depends on the number of single-particle states that overlap at a given site $i$, which is roughly $m$ and is independent of the system size.

In this argument, we have ignored the fact that we are actually interested in localized single-particle states which can have exponential tails, rather than states with strictly local support. It is easily shown that the overlap of a state where these tails have been truncated with the full state approaches one exponentially quickly as the support $m$ of the strictly local states is increased, but this does not imply that the bipartite entanglement matches to the same accuracy. However, it seems plausible that for the eigenstates of local Hamiltonians, the entanglement properties, or at least the scaling of entanglement entropies, does not depend on these tails for $m$ sufficiently large.

We have argued above that when the state of the noninteracting system can be written by filling a set of strictly local single-particle orbitals, the bipartite entanglement entropy is independent of system size and displays an arealaw scaling. Consider now our colloquial definition for an MBL state, which is that it can be transformed to a Slater determinant of localized single-particle states almost everywhere to within some desired accuracy by a finite-depth local unitary circuit. Such a circuit can, by similar arguments as given above, increase the entanglement entropy only by some constant amount depending on its depth. By this definition, a many-body localized state would thus inherit the area law from the corresponding Slater determinant. An important caveat is that using a finite-depth local unitary circuit, we can transform a state of the many-body system to a Slater determinant only to within desired accuracy for local properties. For a rigorous argument, we would have to show that if two eigenstates of a local Hamiltonian are locally nearly identical then their entanglement entropies scale the same way. Although reasonable, this is a non-trivial proposition, and we do not attempt to prove it here. We thus propose the following as a conjecture, which will be true if small errors in the local structure of the state do not lead to errors in the scaling of the von Neumann entropy of the reduced density matrix for a large subset of the system:

Conjecture 1. A many-body localized state $|\psi\rangle$ has an area law for the entanglement entropy, that is for almost all regions of sites $\mathcal{R}$, the entanglement entropy $S_{\mathcal{R}}$ has

$$
S_{\mathcal{R}}<\alpha \partial \mathcal{R}
$$

for some constant $\alpha>0$.

Here, $\partial \mathcal{R}$ denotes the boundary area of $\mathcal{R}$, and

$$
S_{\mathcal{R}}=-\operatorname{Tr} \rho_{\mathcal{R}} \log \rho_{\mathcal{R}} \quad \rho_{\mathcal{R}}=\operatorname{Tr}_{\mathcal{L} \backslash \mathcal{R}}|\psi\rangle\langle\psi| .
$$

This is to be contrasted with a volume law, where $S_{\mathcal{R}} \sim$ Vol $\mathcal{R}$. In one dimension, this means that for a system of 
size $L$ which is cut in a generic location, the bipartite entanglement entropy is constant instead of proportional to $L$. In higher dimensions, the entanglement entropy will scale as $L^{d-1}$ instead of $L^{d}$.

This condition is useful for the following reason. Although Definition 2 is precise, it is difficult to find the necessary local unitary transformation. On the other hand, the entanglement entropy is more readily calculated, given an energy eigenstate. However, our definition may still not be directly applicable as we cannot solve the system in the thermodynamic limit, or in a sufficiently large system to be self-averaging with enough distinct regions $\mathcal{R}$ of different size. In a practical calculation, we therefore have to consider an ensemble of Hamiltonians and compare the entanglement entropies obtained for different system sizes.

We therefore formulate the following criterion that we use to identify a many-body localized phase. We will give a more precise definition in Sec. III D, but for now, we suggest the following more colloquial definition:

If almost all energy eigenstates of a Hamiltonian have an area law for their entanglement entropy for almost all regions, then we will say that the system is in a many-body localized phase.

We will make precise what we mean by "almost all" eigenstates in Def. 3 .

However, this definition is satisfied by many trivial systems, for example systems with only on-site terms or coupling terms that are diagonal in a product basis, such as an Ising model with a longitudinal (instead of the transverse) field. The area law observed for arbitrary eigenstates of such trivial systems is unstable against many perturbations, though. We therefore formulate the following, stricter definition which serves as sufficient and necessary condition for an MBL regime:

A system described by a Hamiltonian $H$ is in a manybody localized phase if almost all eigenstates of

$$
H+\lambda \Phi,
$$

have an area-law for their entanglement entropy for arbitrary perturbations $\Phi$ that are a sum of bounded local operators and for some non-vanishing range of $\lambda$, e.g. for all $|\lambda|<\lambda_{c}$.

We conjecture that this definition excludes systems that have an area law for almost all eigenstates for trivial reasons that should not be considered many-body localization. Thus, it paves the way for using the entanglement entropy to identify many-body localization.

\section{Numerical Calculation of Entanglement Entropy in Energy Eigenstates of a 1D Model of Interacting Electrons in a Random Potential}

We now turn to a numerical analysis of the entanglement entropy in random eigenstates of the system described by Eqn. (2). We calculate the entanglement entropy at the center of the system, i.e. for a block of size $L / 2$. We perform an average over at least 1024 disorder realizations, and for each realization extract the entropy for $L$ randomly-chosen eigenstates. From now on, the quantity $S$ will refer to the entanglement entropy between the left- and right-halves of the system, and we will drop the subscript $\mathcal{R}$ in $S_{\mathcal{R}}$ since $\mathcal{R}$ will always be the left (or, equivalently, the right) half of the system. Occasionally, we will use the notation $S_{c}$ to emphasize that this is the entropy obtained by cutting the system at its center.

Fig. 2 has histograms showing the total number of states that have entanglement entropy $S_{c}$ (y-axis) in the entire ensemble of 1024 systems at each size ( $x$-axis) from $L=4$ to $L=16$. We show results for three strengths of the disorder potential, $W=4,6,8$, and three strengths of the interaction, $V=0,0.4,1.2$. Each histogram also shows the median value of $S_{c}$ (dashed line). Consider first the non-interacting case $V=0$ (top row in Fig. 2), where the system is a noninteracting Anderson insulator. As expected, we observe an entanglement entropy that is largely independent of system size but does depend on the disorder strength since the amount of local contributions to the entanglement entropy depends on the localization length. For $W=6$ and $W=8$, additional peaks at $S \approx \ln (2)$ become visible. These peaks can be traced to a purely local effect - a localized state spread over the two sites straddling the center of the system - which is discussed in Appendix B. However, apart from a small but noticeable number of states that have $S \approx \ln (2)$, almost all states have entropies near or less than the median entropy, which has very weak dependence on system size.

Although our primary interest is the scaling of $S$ with $L$, it is useful to view $\ln (2)$ as a heuristic boundary between lowand high-entropy states. When there are very few states with $S>\ln (2)$, the median entropy does not scale with system size but when a large fraction of the states of the system have $S>$ $\ln (2)$, the median entropy tends to scale with $L$. This is not, by any means, a precise boundary, but it is nevertheless useful to refer to "low-entropy states" and "high-entropy" states, which refers to their entropy relative to $\ln (2)$.

Returning to Fig. 2, we now consider the case of weak interactions and strong disorder, $V=0.4, W=8$. The histogram is very similar to those of the non-interacting case: the median entanglement entropy depends very weakly on system size and most states have entropies near or less than the median entropy. Even for stronger interactions, but strong disorder, $V=1.2, W=8$, the histogram is still similar to the non-interacting case, although one might argue that a slight increase in the median $S_{c}$ with $L$ is visible. But even for this strong interaction, most of the states have very low entropy.

As we decrease the disorder, we find that, for weak interactions and moderate disorder, $V=0.4, W=6$, there are still very few high-entropy states, and the median entropy has weak dependence on $L$. However, for strong interactions and moderate disorder, $V=0.4, W=6$, the number of highentropy states clearly increases with $L$, and the median entropy increases with $L$ in a manner roughly consistent with linear increase.

Finally, we consider the weak disorder case, $W=4$. For weak interactions $V=0.4$, the number of high-entropy states increases with system size, roughly linearly. In this case, in- 

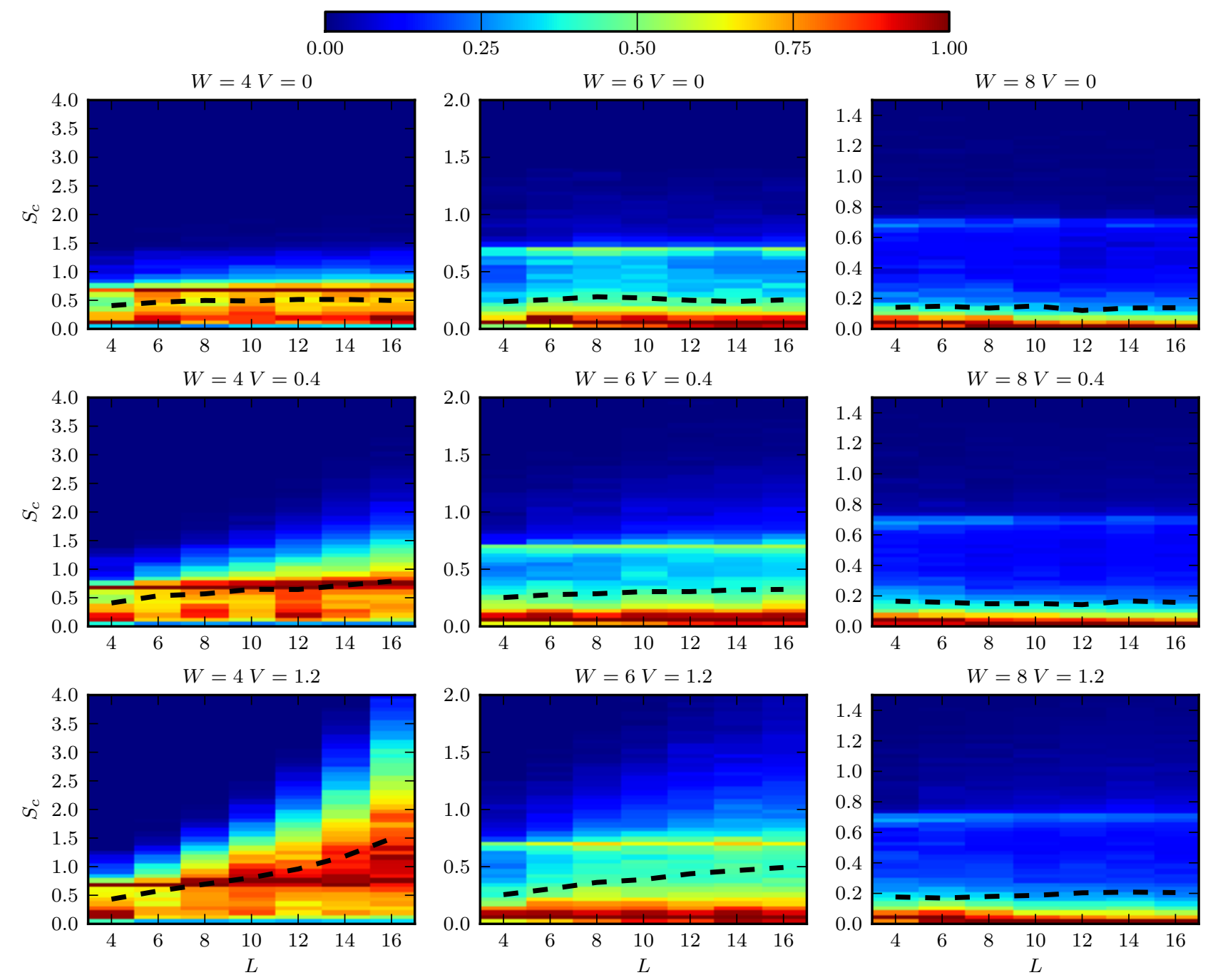

FIG. 2. (Color online) Histogram of the entanglement entropy of random eigenstates of the Hamiltonian (2) at different values of disorder strength and interaction. Dark blue indicates a small density of states at a given $L$ and $S_{c}$, whereas red indicates a higher density. Dashed lines indicate the median value. Results are extracted from 1024 disorder realizations and $L$ randomly chosen states for each realization.

teractions have caused a large fraction of the states to be "delocalized", i.e. to have large entanglement entropy due to entanglement between all parts of the system. This is reflected in the median entanglement entropy, which grows with $L$, but slowly. Finally, when we consider stronger interactions, $V=1.2$, we find that the number of high-entropy states increases linearly with $L$ for $W=4$. The median entropy scales linearly or, perhaps, even faster, although the apparent superlinear growth that sets in at around $L=12$ is probably an increase in the slope from its small $L$ value to its large $L$ value.

The most salient and striking feature of these data is that for the stronger disorder potentials $W=6,8$, the vast majority of states at $V=0.4$ have low entanglement entropy for the system sizes accessible to our simulations. It seems extremely unlikely that this behavior would change for larger system sizes: if the behavior of the system is governed by a large (or divergent) length scale, we would expect a volume law for block sizes smaller than this length scale and a po- tential crossover to an area law beyond this scale; the other situation, i.e. observing an area law on short scales and a volume law at large scales, is very unlikely. However, we cannot exclude the possibility of extremely slow growth.

The histograms in Fig. 2 include states of all energies. However, separating the states by energy does not change the picture very much. High entropy states occur primarily near the center of the spectrum, but even at the center of the spectrum there are states with very low and very large entropy regardless of the strength of interactions. Hence, for the system sizes available to us, no sharply-defined many-body analogue of a mobility edge can be numerically observed for these intermediate values of disorder and interaction strength.

One important feature of this data, which is somewhat masked by the color scale in Figs. 2, is that there are high entropy states at all energies. As may be seen from Fig. 3, these states become more rare as we consider larger and larger entanglement entropies. We suggest the following heuristic pic- 

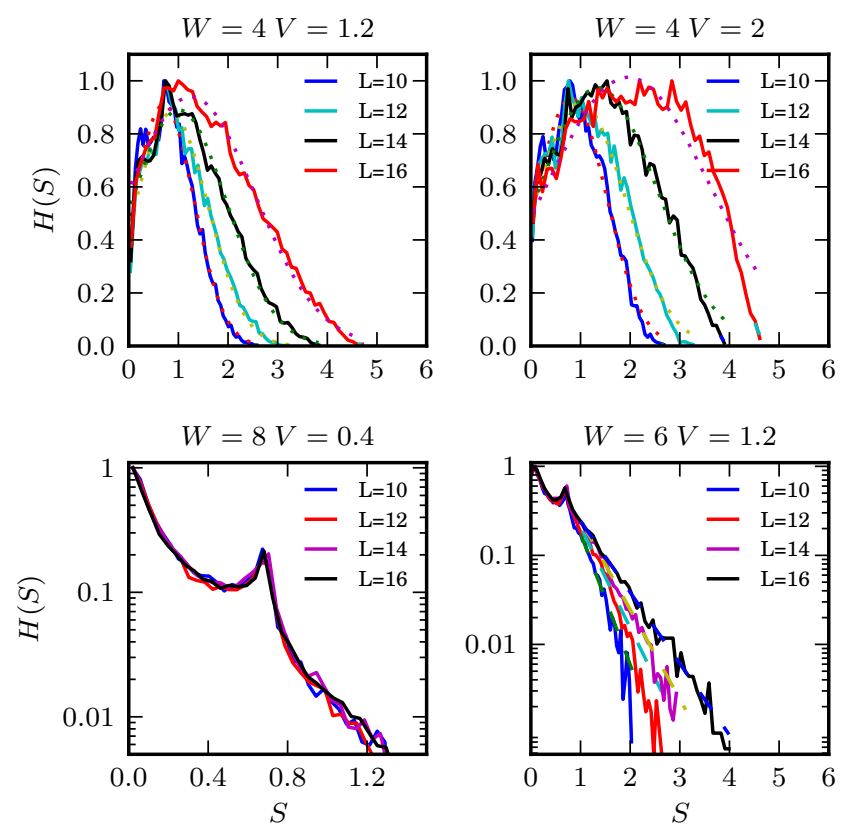

FIG. 3. The number of states $H$ with entropy $S$ for different system sizes and system parameters. Plots in the top row show parameters where the system is entering a metallic regime and the histogram follows (15). In the lower left, the system is clearly in the MBL regime, where the histogram follows (14) with $a$ independent of $L$. In the lower right panel, some drift of $a(L)$ with $L$ is observed, putting the system on the edge of the MBL regime. Dashed lines indicate fits to (14), dotted lines are fits to (15).

ture: the entanglement generally remains small because there are "entanglement bottlenecks". These are places where, for reasons which we examine in more detail below, entanglement cannot be generated in a given state. If we assume a probability $p_{b}$ that a given site is a bottleneck, then a region with entropy $S$ will occur somewhere in the system with probability $p(S) \sim L\left(1-p_{b}\right)^{S}$. The probability that we will obtain this entanglement entropy from an arbitrary cut through the system (e.g. at the middle) is $\sim\left(1-p_{b}\right)^{S}$. This leads to an exponentially-decaying density $H(S)$ of states with entanglement entropy $S$ across the mid-point of the system, $H(S) \sim e^{-S / a}$.

In Fig. 3, we see four different types of behaviors. From the lower left panel of Fig. 3, we see that, for $W=8, V=0.4$, the density of states $H(S)$ with entanglement entropy $S$ indeed decays exponentially, as expected from the above discussion. (Note that there is a peak at $S=\log 2$, as discussed above.) In the upper panels, $H(S)$ clearly does not satisfy the definition of an MBL phase for $W=4, V=1.2$ or 2 , as we discuss below. In the lower right panel, where $W=6, V=1.2$, the situation is unclear. This will be discussed below.

First, however, we examine more quantitatively the extent to which the distribution of entropies $H(S)$ agrees with our expectations. We consider the more general form for a finite-

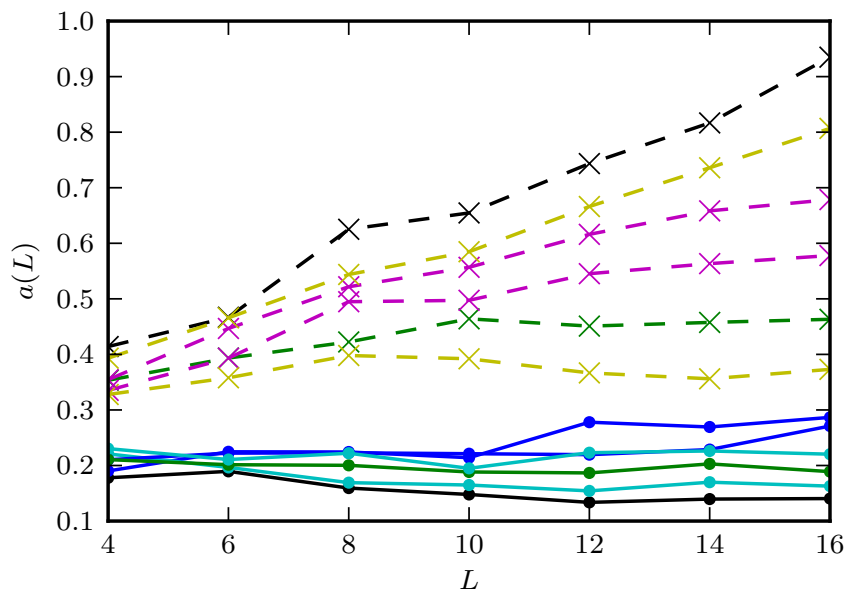

FIG. 4. Coefficient $a(L)$ extracted from fits to Eqn. (14). Solid lines are $W=8$, dashed lines are $W=6$; interaction strength is $V=$ $0,0.4,0.8,1.2,1.6,2$ from bottom to top.

size system:

$$
H(S) \sim e^{-S / a(L)}
$$

In a many-body localized regime, we expect $a(L)$ to approach a finite constant as $L \rightarrow \infty$. For $W=8$, Fig. 4 shows that $a(L)$ is approximately constant over a wide range of interaction strengths $V$, as expected if the system were in an MBL phase in this range. For $W=6, a(L)$ appears be constant for $V=0.4$ (second dashed line from the bottom in Fig. 4) but it increases for larger values of $V$. Finally, for $W=4$ (not shown), $a(L)$ appears to increase for all of the values of $V>0$ studied, with the possible exception of $V=0.2$.

In a regime where the system is metallic but disorder remains strong enough to affect the local physics, we expect that the mean entropy scales with system size and the entropies are normally-distributed around the mean value, so that

$$
H(S) \sim e^{-\left(S-s_{0} L\right)^{2} / \alpha L}
$$

This is observed for weak disorder, $W=4$, and strong interactions, $V=1.2$ and $V=2$, in the upper panels of Fig. 3. The peak of the Gaussian is approximately at the median value of the entropy, which increases linearly with system size $L$. This is somewhat obscured at smaller system sizes because the $\ln (2)$ peak dominates the data. It may be somewhat surprising that we obtain these results even without restricting to states of a single energy because, in a metallic state in thermal equilibrium we expect the entropy density $S / L$ to grow with the energy of the state. However, the histogram is dominated by states near the center of the (many-body) energy spectrum because there are exponentially more of them than there are states in the tails of the spectrum. Therefore, $H(S)$ is nearly indistinguishable from $H(S, E)$, the histogram restricted to an energy window around $E$, if $E$ is near the center of the spectrum. On the other hand, in the band tails $H(S, E)$ looks similar to the MBL regime. 
For intermediate parameters between the MBL and the metallic regimes, we observe a different behavior of $H(S)$. Consider, for example, $W=6$ and $V=1.2$. From Fig. 2, we see that for these parameters the median value of $S$ increases slowly with $L$, consistent with $S \propto L$ but with small proportionality constant. This is reflected in the lower right panel of Fig. 3, where we see that $H(S)$ decays as $H(S) \sim e^{-S / a(L)}$ with $a(L)$ increasing. The coefficient $a(L)$ increases with system size, as may be seen in Fig. 4. Therefore, at least for these system sizes, the system at $W=6$ and $V=1.2$ is qualitatively different from the systems at $W=4$ and $V=1.2$. Although the median entropy grows with system size in both cases, the distribution of entropies is rather different. In the latter case, it appears similar to thermodynamic equilibrium but in the former case, it looks more like a long tail of high entropy states in an MBL system.

In Fig. 4, we can see that for $W=6$ and $V=1.2, a(L)$ grows linearly for small systems and the growth slows down for the largest systems accessible to our simulations. The simplest possibility is that the system is in an MBL phase, and $a(L)$ approaches a finite limit for large enough systems. An alternate possibility is that the system is in a metallic phase, but with low $S / L$. However, it is also possible that the system is in an intermediate phase in which $a(L) \sim L^{\beta}$ at large $L$, with $0<\beta \leq 1$ so that a non-zero fraction of the states of the system have entanglement entropy $S>\kappa L^{\beta}$ for some $\kappa$. This would not be a conventional metallic phase, even if $\beta=1$ (or, more generally, $\beta=d$ in $d$-dimensions). Rather, it is a phase in which an $\mathcal{O}(1)$ fraction of the states has low entanglement entropy and an $\mathcal{O}(1)$ fraction has large (though possibly sub-linear) entanglement entropy. We call this a "long tail" regime, in which the median entropy increases with system size - possibly even linearly - but the system is not in an equilibrium metallic phase because an $\mathcal{O}(1)$ fraction of the states of the system have $\mathcal{O}(1)$ entanglement entropy, even if we restrict attention to energies near the center of the spectrum.

\section{Definition of a many-body localized phase}

In this section, we condense the above considerations about the statistical distribution of entanglement entropies into a concise definition for a many-body localized phase.

Let us revisit our heuristic picture for the entanglement bottlenecks. These can occur for two reasons: There can be disorder realizations in which the on-site potential is very large or very small at a given site, thereby effectively cutting the system there. Conversely, there can be disorder realizations in which the on-site potential is nearly the same over clusters of several sites, which is analogous to the two-site clusters that cause the $\ln (2)$ peak discussed previously and in Appendix B. This leads to the absence of bottlenecks in that cluster of sites. A second reason why bottlenecks could occur is the particular choice of state. For a fixed disorder realization, there may be arrangements of the particles - possibly of very high energy - which effectively cut the system in two, thereby forming a bottleneck. Of course, these two mechanisms are not indepen- dent. Regardless of the ultimate cause of the bottleneck, the entanglement entropy across it would be low because particle and correlations cannot propagate through a bottleneck.

Let us now consider a very large system $L$, where most of the states are MBL states. We have argued that the probability of finding entropy $S$ in such a system for a generic cut is $H(S) \sim e^{-S / a}$; cuts where $S / a \gg 1$ are therefore unlikely. One could, however, also ask: what is the probability of finding some cut of the system for which the entanglement entropy is $S$ ? This clearly follows $\tilde{H}(S) \sim L e^{-L / a}$, and hence only cuts where $S / a \gg \ln L$ are rare; in other words, the maximum entanglement entropy found in the system diverges logarithmically, while the median entanglement entropy saturates to an area law. This behavior is observed in the data shown in Fig. 5. However, our arguments also suggest that for a given state of a very large system, the distribution of entanglement entropies obtained by cutting in different ways has a finite variance, i.e. while the maximum may diverge, the median (and other quantiles) of the distribution remain finite. In other words, as the thermodynamic limit is approached, the cuts that lead to a divergent entanglement entropy become a set of measure $(\ln L) / L$. Relating this back to our Def. 2, we can expect that using a finite-depth unitary transformation, an MBL state can be transformed into a Slater determinant of localized single-particle orbitals everywhere except in an exponentially-small fraction of the system.

Note that this is very different from the case of singleparticle localization. In the single-particle case, there may be regions of size $\ln L$ in which the on-site potential is nearly constant over the entire region. However, this is not sufficient for a state with localization length $\sim \ln L$. As we consider larger and larger regions, the potential must get flatter and flatter over the entire region in order for a delocalized state to occur. Thus states with large localization length occur only occur for disorder configurations of measure zero in the probability distribution of the on-site disorder ${ }^{20}$. However, in the case of MBL, the on-site potential need not be fine-tuned to be within some set of configurations of measure zero. There could, instead, be arrangements of the particles that allow entanglement to build up even for more generic disorder configurations.

With the preceding considerations in mind, we define a many-body localized phase as follows.

Definition 3. An infinite system described by some Hamiltonian $H$ is in an $M B L$ phase if, for any $f, \epsilon, k$ there exist finite real numbers $D, b, c$ such that for any $d>D$, all energy eigenstates are MBL states with localization depth $\leq d$ to accuracy $(\epsilon, k)$ on a volume fraction $f$ of the system, except for a fraction of states $<b e^{-c d}$.

Note that in a many-body localized system, generically no mobility edge can be observed for the following reason: In a non-interacting Anderson insulator, localized and extended single-particle states cannot coexist at the same energy - if there were both a localized and an extended single-particle state at the same energy, it would require fine-tuning for them to have vanishing matrix elements. Small changes in the Hamiltonian would mix them, causing both states to become 


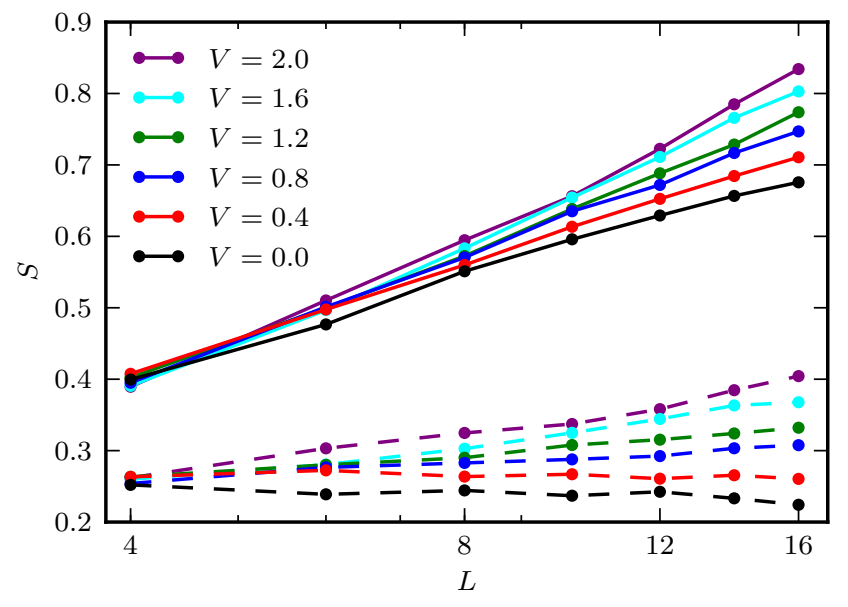

FIG. 5. Disorder-averaged entropy (i) for the cut at the center of the system (dashed lines) and (ii) for the cut with the maximum entropy in each disorder realization (solid lines) for $W=6$ on a semilogarithmic scale. While an area law is observed for the center cut for weak enough interactions, a logarithmic divergence of the entropy for the maximum entropy cut is found for these interaction strengths. For strong enough interactions, both diverge with a volume law.

extended. In the many-body system, on the other hand, lowand high-entropy states can exist at the same energies because the matrix elements between these many-body states could vanish due to an orthogonality catastrophe: they may differ in the arrangements of an extensive number of particles so that the matrix elements between them for any local Hamiltonian would vanish.

\section{TOPOLOGICAL ORDER AT NON-ZERO EXCITATION ENERGY DENSITY AND SELF-CORRECTING QUANTUM MEMORY}

\section{A. Overview}

A system is in a topological phase in its ground state if it satisfies the following definition: On a manifold $\mathcal{M}$, it has a set of orthonormal ground states $|a\rangle, a=1,2, \ldots, N_{\mathcal{M}}$. The degeneracy $N_{\mathcal{M}}$ depends only on the topological configuration of the system, e.g. genus, number of boundaries, and boundary conditions. These states are separated from the rest of the spectrum by an energy gap $\Delta$ that remains non-zero in the limit that the system size $L \rightarrow \infty$. Furthermore, for any local operator $\phi$,

$$
\langle a|\phi| b\rangle=C \delta_{a b}+\mathcal{O}\left(e^{-L / \xi}\right)
$$

where $C$ is a constant independent of $a, b ; L$ is the system size; and $\xi$ is the correlation length of the system, which is finite in the limit $L \rightarrow \infty$. The Hamiltonian is a sum of local operators, so the energy splitting between the $\mathcal{M}$ ground states vanishes exponentially with the system size.

In many situations, a topological phase can equivalently be defined as a gapped phase which cannot be transformed to a

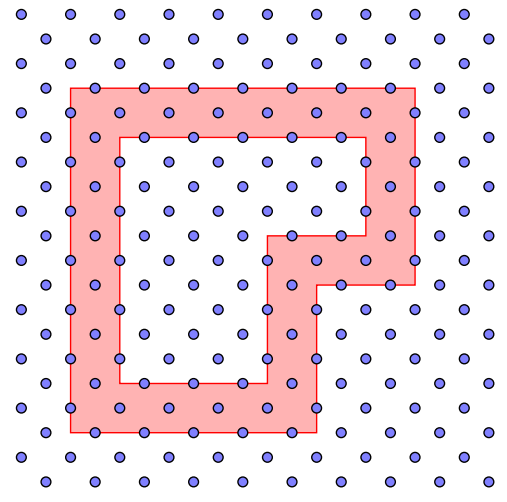

FIG. 6. Fattened Wilson loop operator $W_{a}(\mathcal{C})$.

local product state by a local unitary transformation of finite depth. For a symmetry-protected topological phase, which is not our primary focus here, one has to augment this definition by requiring that the unitary transformation leave the symmetries untouched. However, as was shown in Ref. 49, there is a subtle difference between these definitions when considering disordered systems with ground states that have a non-zero density of localized quasiparticles: these will generally not satisfy the latter definition since the quasiparticles effectively partition the system into finite blocks that can be transformed to a product. However, such a system could still satisfy the first definition given above.

A similar situation is encountered for systems at non-zero temperature in two dimensions, where a non-zero - albeit exponentially small in $T / \Delta$ - density of quasiparticles is thermally induced. Such a system clearly violates the second definition and it is not immediately clear how to extend the first definition to excited states at finite energy density. However, it is conceivable that topological order survives even in states of finite energy density above the ground state if the quasiparticles are many-body localized. In this section, we seek to find a definition of a topological phase that encompasses all these scenarios. Similar ideas were recently put forward in Ref. 26. We will largely rely on the area law as a definition of a many-body localized regime.

Following Ref. 50, we will define a topological phase (in its ground state, for now) in terms of a "zero-law" for Wilson loop operators. At an intuitive level, the definition is the following: We define appropriate "fattened" Wilson loop operators $W_{a}(\mathcal{C})$ associated to curves $\mathcal{C}$ (cf Fig. 6) and quasiparticle types $a$ (see, for instance, Ref. 51 and references therein). These operators will be defined explicitly below. In a topological phase in its ground state, this Wilson loop operator will have the following properties: When measured on the sphere or plane, such operators have ground state expectation values $\left\langle 0\left|W_{a}(\mathcal{C})\right| 0\right\rangle \neq 0$ that are independent of the perimeter $P_{\mathcal{C}}$ of $\mathcal{C}$. On manifolds with non-trivial topology, such operators can be used to distinguish the different degenerate ground states. Depending on the curve $\mathcal{C}$ and quasiparticle type $a$, Wilson loop operators can have expectation values that are different for different ground states or can have non-vanishing matrix elements between different ground states. When measured in 
a non-topological phase, on the other hand, the expectation values of such Wilson loop operators will decay as $e^{-a P}$ for some constant $a$.

The reason that we need to consider "fattened" Wilson loop operators is that, even in the ground state of a topological phase, virtual quasiparticle pairs will be created and one member of the pair can fluctuate between the interior and exterior of the region bounded by $\mathcal{C}$. If the Wilson loop operators are "fattened" so that they have a width that is larger than the length scale for such fluctuations, then virtual quasiparticle will not cause $\left\langle W_{a}(\mathcal{C})\right\rangle$ to wash out.

Let us now consider the case of a topological phase at finite temperature, i.e. at finite energy density above the ground state. In this setting, thermally excited and mobile quasiparticles will hop in and out of the loop, washing out $\operatorname{Tr}\left(e^{-\beta H} W_{a}(\mathcal{C})\right)$ and leaving behind a strong dependence on the perimeter of the curve $\mathcal{C}$. If, however, quasiparticle excitations are localized in the energy eigenstate $|E\rangle$, this may be prevented: Suppose that we have a topological phase with a large gap. Then, since the gap is large, we can, with negligible effect on the ground state, tune the Hamiltonian so that the quasiparticles have a large effective mass and we can tune the disorder so that it has a large effect on the quasiparticles above the gap. If quasiparticle excitations are many-body localized in this limit, which seems ideally suited for it to occur, then excited quasiparticles will be unable to move. Therefore, they will be unable to wash out $\langle E|W(\mathcal{C})| E\rangle \sim$ const.

To define topological order in the ground state, we could exploit either the ground-state degeneracy or Wilson operators. While they are equivalent, the former is typically more convenient since it is not always obvious how to define the appropriate Wilson loop operators. However, as was discussed above, it is very difficult to use degeneracy as a criterion since it may be hard to identify the correct correspondence between degenerate states in different topological sectors since the density of states at energies much above the spectral gap is always exponentially large. However, as pointed out by Huse et al. ${ }^{26}$, there are scenarios where such an approach can be applied.

In essence, the Wilson construction we describe here could allow us to identify topological multiplets in the midst of a very high density of states. In the case of a Hamiltonian whose clean limit we understand, it could be possible to construct these operators and, by entering the regime noted above, test the possibility of topological order stabilized by many-body localization.

\section{B. Example: Toric Code}

Consider the perturbed toric code Hamiltonian ${ }^{52}$ with random couplings:

$$
\begin{aligned}
H_{\mathrm{TC}}=-\sum_{v} J_{e}(v) A(v)- & \sum_{p} J_{m}(p) B(p) \\
& -h \sum_{i} \sigma_{i}^{z}-\tilde{h} \sum_{i} \sigma_{i}^{x}
\end{aligned}
$$

Here, $A(v)$ and $B(p)$ are defined by:

$$
A(v)=\prod_{j \in \operatorname{vertex}(v)} \sigma_{j}^{z}, \quad B(p)=\prod_{j \in \operatorname{plaquette}(p)} \sigma_{j}^{x}
$$

and $J_{e}(v)$ and $J_{m}(p)$ are independent random variables chosen uniformly from $J_{e}(v) \in\left[J_{0}^{e}-W, J_{0}^{e}+W\right]$ and $J_{m}(p) \in$ $\left[J_{0}^{m}-\tilde{W}, J_{0}^{m}+\tilde{W}\right]$. The magnetic fields $h, \tilde{h}$ create pairs of, respectively, magnetic and electric excitations and move these excitations. In the limit that $h, \tilde{h} \ll W, \tilde{W} \ll J_{0}^{e}, J_{0}^{m}$, the ground state is essentially the ground state of the toric code and the gap to all excitations is very large ${ }^{53-58}$. However, at energies larger than the gap, excitations will be localized: although $h, \tilde{h}$ could allow them to move, the disorder $W$ is so much larger that both electric and magnetic particles will get trapped in regions where $J_{e}(v) \approx J_{0}^{e}-W$ or $J_{m}(p) \approx J_{0}^{e}-W$, respectively. If many-body localization occurs, then electric and magnetic particles will be unable to move, even in states with non-zero energy densities and in the presence of perturbations such as the following, which may be viewed as an interaction between quasiparticles:

$$
H_{\mathrm{int}}=V \sum_{\left\langle v, v^{\prime}\right\rangle} A(v) A\left(v^{\prime}\right)+\tilde{V} \sum_{\left\langle p, p^{\prime}\right\rangle} B(p) B\left(p^{\prime}\right) .
$$

$V$ is an interaction between electric particles on neighboring vertices while $\tilde{V}$ is an interaction between magnetic particles on neighboring plaquettes.

For $h, \tilde{h}=0$, the Wilson loop operators

$$
W_{m}\left(\mathcal{C}_{D}\right)=\prod_{i \in \mathcal{C}_{D}} \sigma_{i}^{z}, \quad W_{e}(\mathcal{C})=\prod_{i \in \mathcal{C}} \sigma_{i}^{x}
$$

have expectation value 1 in the ground state, i.e. $\left\langle 0\left|W_{m}\left(\mathcal{C}_{D}\right)\right| 0\right\rangle=1,\left\langle 0\left|W_{e}(\mathcal{C})\right| 0\right\rangle=1$ for any contractible closed curves $\mathcal{C}$ on the lattice and $\mathcal{C}_{D}$ on the dual lattice. However, for $h, \tilde{h} \neq 0$, virtual excitations of pairs of quasiparticles straddling the curves $\mathcal{C}, \mathcal{C}_{D}$ will cause these expectation values to vanish for large loops as $\left\langle 0\left|W_{m}\left(\mathcal{C}_{D}\right)\right| 0\right\rangle \sim e^{-a P_{\mathcal{C}_{D}}}$ and $\left\langle 0\left|W_{e}(\mathcal{C})\right| 0\right\rangle \sim e^{-a P_{\mathcal{C}}}$, where $P_{\mathcal{C}}$ is the perimeter of a the curve $\mathcal{C}$. Thus, we must consider 'fattened' Wilson loop operators. Consider, for instance,

$$
W_{m}^{F}\left(\mathcal{C}_{D}\right)=\prod_{i \in \mathcal{C}_{D}} \frac{1}{2 n+1} \sum_{j=-n}^{n} \sigma_{i+j_{\|}}^{z}
$$

where $i+j_{\|}$is the link of the lattice that is $j$ spacings away from link $i$ in the direction of link $i$. For $n$ much larger than the typical separation of a virtual pair of electric excitations (which is $\sim \tilde{h} / J_{0}^{e}$ for small $\tilde{h}$ ), $\left\langle 0\left|W_{m}^{F}\left(\mathcal{C}_{D}\right)\right| 0\right\rangle>0$ for $h>0$ but $T=0$. In other words, the topological phase survives small quantum fluctuations at zero temperature, as can be diagnosed with fattened Wilson loop operators.

Now consider non-zero temperature. For vanishing disorder, $W, \tilde{W}=0$, and non-zero temperature $T>0$, there will be a non-zero density of thermally-excited quasiparticles. For large loops $\mathcal{C}_{D}, \operatorname{tr}\left(e^{-\beta H} W_{m}^{F}\left(\mathcal{C}_{D}\right)\right) \sim e^{a P_{\mathcal{C}_{D}}}$ because the statistical average will include nearly equal numbers of states 
with even and odd numbers of quasiparticles within each loop $\mathcal{C}_{D}$.

Suppose now that an MBL phase occurs in this model in the regime $h, \tilde{h} \ll W, \tilde{W} \ll J_{0}^{e}, J_{0}^{m}$. Then, in an MBL eigenstate of energy-density $\varepsilon$, there will be a non-zero density $\sim \varepsilon / J_{0}^{e, m}$ of localized quasiparticles. If this density is low and the localization length is short, we can choose curves $\mathcal{C}_{D}$ and 'fattened' Wilson loop operators $W_{m}^{F}\left(\mathcal{C}_{D}\right)$ that avoid these quasiparticles by passing between them. (We will say a bit more about avoiding quasiparticles in the next paragraph) Then, for any particular realization of the disorder, we have $\left\langle\varepsilon\left|W_{m}^{F}\left(\mathcal{C}_{D}\right)\right| \varepsilon\right\rangle \sim$ const., although its precise value will depend on the particular curve and how many localized quasiparticles it encloses. This is the analog for non-zero energy density of the situation that occurs for two quasiparticles, which was studied by Stark et $a l^{59}$. On the other hand, if we average over disorder realizations or over locations of the curve within the system, we will obtain zero.

If we know a priori where the localized quasiparticles are, then we can simply choose a curve that avoids them, assuming that the density of quasiparticles $n_{\mathrm{qp}}$ satisfies $n_{\mathrm{qp}} \xi^{2} \ll 1$, where $\xi$ is their localization length. On the other hand, if we do not know where the quasiparticles are located and simply place a curve $\mathcal{C}_{D}$ on the dual lattice at random, then it has a probability $\sim n_{\mathrm{qp}} \xi L_{\mathcal{C}_{D}}$ of intersecting one of these localized quasiparticles, according to Crofton's formula. So long as $n_{\mathrm{qp}} \xi L_{\mathcal{C}_{D}} \ll 1$, which puts constraints on the energy density, then there will be a range of lengths $L_{\mathcal{C}_{D}} \ll$ $1\left(n_{\mathrm{qp}} \xi\right)$ over which even a randomly chosen curve $\mathcal{C}_{D}$ will give $\left\langle\varepsilon\left|W_{m}^{F}\left(\mathcal{C}_{D}\right)\right| \varepsilon\right\rangle$ independent of the size of the loop with high probability.

We can make this more concrete by specializing to the case in which $J_{0}^{e}=\infty$ so that there are no electric particles, only magnetic ones. In a generic energy eigenstate, there will be a non-zero density of magnetic particles. However, if they are many-body localized, they will be essentially frozen into some locations so that a Wilson loop operator $W_{e}(\mathcal{C})$ can have a non-vanishing expectation value for a curve $\mathcal{C}$ that avoids them. In the absence of electric particles, we can perform a duality transformation from the toric code, which is a $\mathbb{Z}_{2}$ gauge theory, to the Ising model. We introduce a new set of spins $\mu_{p}$ located at the centers of the plaquettes. They are defined by

$$
\begin{aligned}
& \mu_{p}^{x}=B(p)=\prod_{j \in \operatorname{plaq}(p)} \sigma_{j}^{x} \\
& \mu_{p}^{z}=(-1)^{n_{p}}
\end{aligned}
$$

where $n_{p}$ is the number of times that $p$ has been flipped, counting from an arbitrary fixed reference state so that $\sigma_{i}^{z}=\mu_{p}^{z} \mu_{p^{\prime}}^{z}$ where $p$ and $p^{\prime}$ share the edge $i$. The Hamiltonian now takes the form

$$
\begin{array}{r}
H=-\sum_{p} J_{m}(p) \mu_{p}^{x}-h \sum_{\left\langle p, p^{\prime}\right\rangle} \mu_{p}^{z} \mu_{p^{\prime}}^{z} \\
=-\sum_{p} J_{0}^{m} \mu_{p}^{x}-h \sum_{\left\langle p, p^{\prime}\right\rangle} \mu_{p}^{z} \mu_{p^{\prime}}^{z} \\
-\sum_{p}\left[J_{m}(p)-J_{0}^{m}\right] \mu_{p}^{x}
\end{array}
$$

This is a transverse field Ising model with an average transverse field $J_{0}^{m}$ and an additional random component $J_{m}(p)-$ $J_{0}^{m}$ in the range $[-W, W]$.

When the transverse field Ising model is disordered, (a fattened version of) the Wilson loop operator $W_{e}(\mathcal{C})$ will have non-vanishing expectation value. When it orders, the spins $\sigma_{i}^{z}$ also order, and $W_{e}(\mathcal{C})$ satisfies a perimeter law. Equivalently, when the spins $\mu_{p}^{z}$ order, a domain wall between upand down-spins has an energy cost proportional to its length. However, if topological order (according to our definition) is present in some eigenstate, then the dual spins will not be ordered. A trivial case of this occurs in the $h=0$ limit. Magnetic quasiparticles cannot move because there is no hopping term for them. In the dual transverse field Ising model, there is no Ising interaction between spins, so an energy eigenstate at finite energy density simply has a non-zero density of $\mu_{p}^{x}=-1$ dual spins. But since there are no spins fluctuating between $\mu_{p}^{x}=1$ and $\mu_{p}^{x}=-1$, the correlation function $\left\langle\mu_{p}^{z} \mu_{p^{\prime}}^{z}\right\rangle$ vanishes for $p \neq p^{\prime}$. On the other hand, if $h \neq 0$ in the absence of disorder, then the $\mu_{p}^{x}=-1$ magnetic particles can move and, consequently, the bipartite entanglement entropy will have a volume law corresponding to a gas of bosons. As a result, even fattened versions of the Wilson loop operator $W_{e}(\mathcal{C})$ will have perimeter laws. However, if the $\mu_{p}^{x}=-1$ magnetic particles are many-body localized for $h \neq 0$ and $W \neq 0$, then the bipartite entanglement entropy of the transverse field Ising model at non-zero energy density will have an area law.

We will call a system that realizes this scenario a topological phase at non-zero energy density, which we will make more precise elsewhere ${ }^{60}$. The basic idea is that when the situation described in the previous paragraph holds, we can measure Wilson loop operators - which could be done in a real system with interferometery experiments (see Ref. 51 and references therein) - and thereby determine where the localized quasiparticles are. Once this is done, we can excite further quasiparticles and braid them. The outcome of such braiding processes will be invariant under small deformations of the quasiparticle trajectories, namely deformations that do not cross localized quasiparticles.

\section{Self-correcting quantum memories}

We now turn to the related, but distinct, problem of selfcorrecting quantum memories. While the question of the stability of a topological phase at non-zero energy density involved the characterization of eigenstates of the system above the spectral gap, the question of stability of self-correcting quantum memories relies on a characterization of the dynamics of the system at long times. The effect of disorder on the stability of quantum memories has previously been discussed for the toric code $\mathrm{e}^{59,61}$ and a one-dimensional Majorana chain $^{62}$, and it was found that disorder can, under the right circumstances, improve the stability of quantum memories. To pose the question, let us suppose that we have quantum information encoded in a physical system. We shield the system as well as we can, but there will always be some rate, however 
small, at which external perturbations such as cosmic rays will pass through our shielding and cause a transition of the system into some other state. If the system is self-correcting, then it means that the quantum information encoded in the state - but not necessarily other properties of the state - is unaffected at long times; more precisely, we will require the error rate to be exponentially-small in the system size.

Consider, for the sake of concreteness, the Toric code in four dimensions $^{63}$, which is an example of a topological phase that survives at $T>0^{64}$. Its stability at $T>0$ is due to the fact that the quasiparticles are string-like excitations which have a string tension. Consequently, an energy proportional to the linear size of the system is required to create a long-enough string to change the topological sector of the system. Let us suppose that the system is initially in some state in the $N_{\mathcal{M}^{-}}$ dimensional ground state subspace of the full Hilbert space. Every time a high-energy photon enters the system, there is some probability that it creates a pair of small string loop excitations. The system, which is in a higher-energy state in the same topological sector, can now relax back to the ground state subspace by pair-annihilating the small string loops and emitting a photon. In order for an error to occur, one of the string loops must wrap around the system before they annihilate. However, it costs energy to increase the length of a string loop. This energy must be supplied by another photon. If the rate at which photons enter the system is sufficiently small, then the system will almost surely relax back before the string can grow. There is a very small probability $p$ for the string loop to grow larger as a result of a second photon before the error has been relaxed away; the probability that a series of such events allows a string to grow to length $L$ is $p^{L}$. Therefore, if this scenario is correct, then the probability that an error occurs is exponentially-small in the system size, i.e. the system is a self-correcting quantum memory.

Consider, in contrast, a clean topological phase in two dimensions ${ }^{65}$, which is known to be unstable to any non-zero temperature. Suppose an incoming photon is absorbed by the system and a quasiparticle-quasihole pair is created. Then, the quasiparticle-quasihole pair could annihilate, emitting a photon. But even if the probability for this is large, there will be some non-zero probability for the quasiparticle and quasihole to move apart from each other rather than immediately annihilate. Since there is no long-distance force holding the quasiparticle and quasihole together, once they move apart, they can move around independently. Eventually, they will meet again and annihilate, but the probability for the difference between the quasiparticle and quasihole trajectories to be a loop encircling the system decreases as a power of the system size $L$. Therefore, even at $T=0$, just a single incoming photon will cause an error with a probability that decreases as a power of the system size (see, e.g., Ref. 66).

Now suppose that the system is dirty and, furthermore, that all quasiparticle and quasihole states are many-body localized. A single incoming photon can create a quasiparticle-quasihole pair, but the quasiparticle and quasihole cannot move on their own and eventually the pair will annihilate and emit a photon. Even if this takes a long time, a single photon cannot lead to an error since the quasiparticle and quasihole can't move suf- ficiently far away to change the information encoded in the quantum state, and hence. If, on the other hand, a second photon impinges on the system before the quasiparticle-quasihole pair annihilates, it could provide one of them with sufficient energy to hop into another localized state. Provided that an external source pumps energy into the system at a constant and sufficiently high rate, the quasiparticle and quasihole may execute a random walk and move far apart. In a finite system, they will eventually meet again and annihilate, but there will be a non-zero probability for the difference between the quasiparticle and quasihole trajectories to be a loop which encircles the system, leading to a change of the information encoded in the system. To summarize, although a single incoming photon cannot cause an error, a non-zero rate of incoming photons can cause errors with probability inversely proportional to a power of $L$ even in a many-body localized system. Since this is not an exponential, it is not a self-correcting quantum memory.

\section{DISCUSSION}

A disorder-driven metal-insulator transition is, seemingly, a transition in the purely dynamical properties of a system. However, single-particle localization can be diagnosed from the properties of an individual wavefunction. For instance, the single-particle eigenstate at a given energy can be computed by the transfer matrix method, and the properties of the state can be deduced from the eigenvalues of the transfer matrix - the system is localized when there is a gap between the two lowest eigenvalues. In this paper, we suggest a definition of many-body localization in terms of individual energy eigenstates. We define a many-body localized state as one which can be transformed almost everywhere into a Slater determinant of localized single-particle states by finite-depth local unitary transformations, to within desired accuracy.

One important potential consequence, which we conjecture to follow from our definition of an MBL state, is that the entanglement entropy of a block within the system scales as the surface area of that block. A many-body localized system hence has the highly unusual property that nearly all eigenstates display an area law. Equivalently, the interior of a region is only correlated with the exterior through local correlations at the boundary.

We find clear evidence for two regimes in a 1D system of spinless fermions in a random on-site potential. For weak interactions, the entanglement entropy is independent of the system size (which is an area law in a $1 \mathrm{D}$ system). For sufficiently large interactions, the entanglement entropy is proportional to the system size. The former is not quite a proof that the system is many-body localized, according to our definition. However, the other known examples of states with area-laws in excited states are not likely to be realized in this context. Therefore, we take our results, summarized in Fig. 2, as a strong indication that there is a weak-interaction regime in which the system's eigenstates are many-body localized such that they can be transformed into a Slater determinant of localized singleparticle states by a finite-depth local unitary transformation.

Our physical picture is that, in an MBL phase, entangle- 
ment bottlenecks emerge, which prevent an extensive entanglement entropy from developing. This leads to an area law but, assuming that the probability for an entanglement bottleneck to occur at a given site is less than 1, it also implies that there will be an exponentially-decaying density of states with high entropies even in the regime that we identify as the MBL phase. Our data is consistent with this and further suggests the interesting possibility of a regime in which the median entropy increases with system size as a result of ever lengthening tails in $H(S)$, as in the lower right panel of Fig. 3 and the upper curves in Fig. 4. This implies a broadening distribution of entropies, rather than a narrow distribution about an increasing mean, as we find for the metallic states in the upper panels of Fig. 3.

Our definition and our calculations focus on the entanglement entropy of energy eigenstates. This shows very different behavior than the entanglement entropy that is generated by the dynamics of a system that is prepared in an initial local product state and then subjected to time evolution under the full, possibly interacting, Hamiltonian. For clean systems, it has been shown that the entanglement entropy can at most grow linearly in time $e^{21,67}$ and approaches a volume law for long times. In contrast to this, Burrell and Osborne ${ }^{19}$ have shown that the entanglement growth in a non-interacting, localized system can be at most logarithmic with time. Several authors ${ }^{13-16}$ have studied the entanglement growth for interacting disordered systems numerically or using a dynamical real-space renormalization approach and have found that the entropy grows logarithmically after an initial delay, but ultimately approaches a volume law at large enough times. Hence, for both a localized and a delocalized system, the entropy approaches a volume law at very long times, but only the saturation value is different between the two cases: in the localized phase, it is smaller than in an ergodic phase. The entanglement entropy of an eigenstate, meanwhile, appears to show a very dramatic difference between many-body localized regime and a metallic one: the entanglement entropy satisfies an area law in one case and a volume law in the other.

According to our definition, if we look at a subsystem of a large system, it does not look like a system in thermal equilibrium. For instance, its entropy will not be extensive, which is an indication that it is not exploring all of the available states at that energy. This also has dramatic consequences for topological order in highly-excited states. If excited quasiparticles - which would ordinarily destroy topological order - cannot move, then one might expect that topological order can survive. In this manuscript, we have defined topological order at finite temperature in terms of the expectation values of 'fattened' Wilson loop operators ${ }^{68}$. Such an operator can avoid localized quasiparticles and give a constant determined by the number of quasiparticles enclosed, rather than a perimeter law. A 2D system at non-zero energy density exhibiting topological order according to this definition would share many properties with the ground state of a topological phase in a disordered system with a non-zero density of localized quasiparticles, but it does not give a self-correcting quantum memory.

\section{ACKNOWLEDGMENTS}

We thank David Huse for his comments on an earlier draft of this paper and his careful explanation of his recent work. We also have the pleasure of acknowledging useful discussions with Joel Moore, Matt Hastings, Michael Freedman, Bryan Clark, Kirill Shtengel, and Joseph Rudnick. C.N. has been partially supported by the DARPA QuEST program and AFOSR under grant FA9550-10-1-0524. Simulations were performed using the ALPS libraries ${ }^{69}$.

During the final stages of the preparation of this manuscript, we became aware of Refs. 18 and 36, where related ideas are discussed.

\section{Appendix A: Localized Single-Particle States and Finite-Depth Unitary Transformations}

\section{Localized Single-Particle States}

In Section III we have made extensive use of 'localized single-particle states'; here, we give them a precise definition. By 'localized single-particle states', we mean normalized single-particle wavefunctions $\phi(x)$ that decay exponentially at long distances. This can be made more precise by demanding that if $\phi(x)$ is a localized single-particle state then for any $\epsilon>0$, there is a function $\chi(x)$ that satisfies the following conditions. (1) $\chi(x)$ is normalized:

$$
\left|\int d^{d} x \chi^{*}(x) \chi(x)\right|=1
$$

(2) The overlap between $\chi(x)$ and $\phi(x)$ satisfies:

$$
\left|\int d^{d} x \chi^{*}(x) \phi(x)\right|>1-\epsilon .
$$

(3) $\chi(x)$ has compact support $\mathcal{R}$ of linear size $L_{\mathcal{R}}(\epsilon)$ satisfying $\lim _{\epsilon \rightarrow 0}\left[\epsilon^{\alpha} L_{\mathcal{R}}(\epsilon)\right]=0$ for all $\alpha>0$.

We now give a definition of a finite-depth local unitary circuit:

Definition 4. An $m$-local unitary circuit of depth $D$ is a unitary operator

$$
U=\prod_{i=1}^{D} \prod_{j=0}^{m-1}\left(U_{j}^{i} \otimes U_{m+j}^{i} \otimes U_{2 m+j}^{i} \otimes \ldots\right),
$$

where $U_{n}^{i}$ is a unitary operator that acts on at most $m$ consecutive sites starting at site $n$.

\section{Appendix B: $\log (2)$ peak in $H(S)$}

To investigate the nature of the additional peaks observed in the histogram $H(S)$ at $S \approx \log 2$, we have plotted in Fig. 7 histograms of the entanglement entropy at the center of the system against the difference between the on-site potentials 


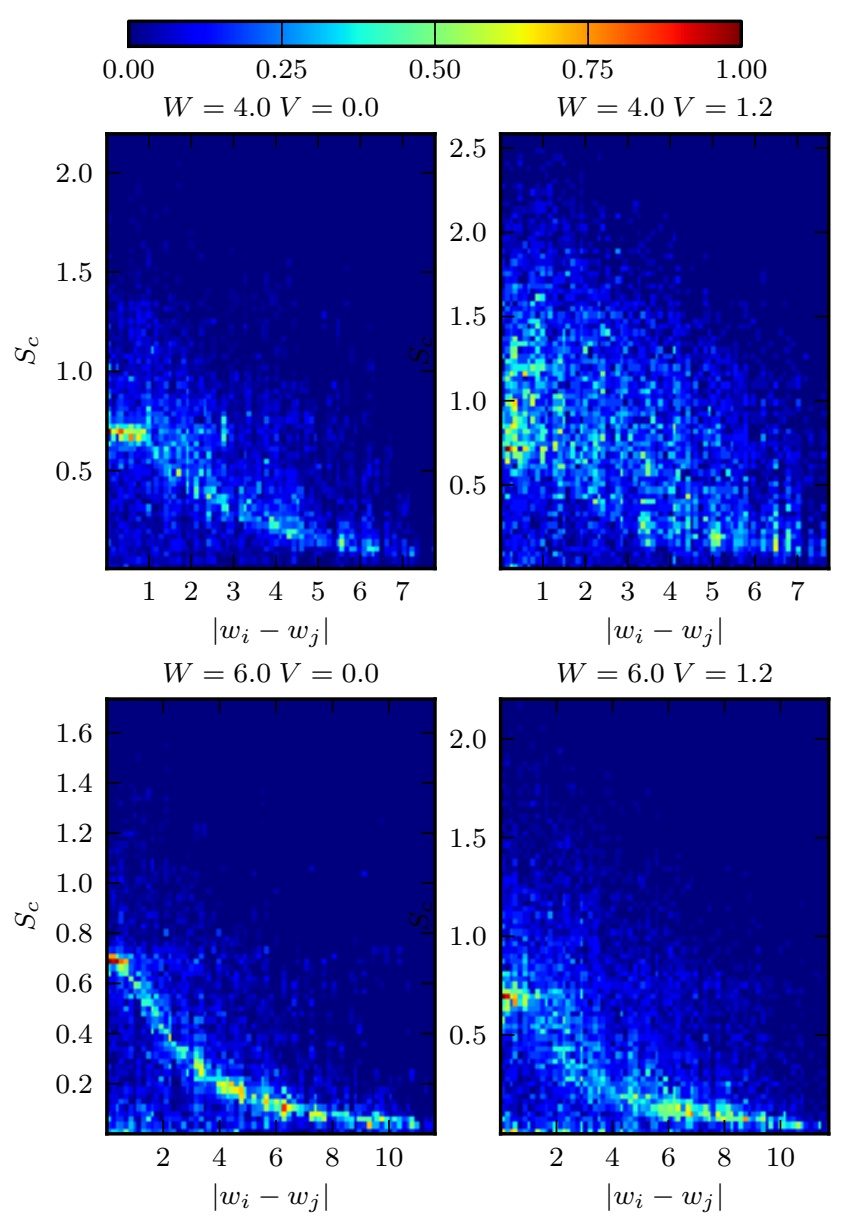

FIG. 7. (Color online) Histogram of the entropy at the center of the system $S_{c}$ vs the difference in on-site potential between the two sites $i, j$ adjacent to the cut. Darker red colors indicate peaks in the histogram. The figure demonstrates that the additional peak at $S_{c}=$ $\log 2$ observed in the entropy data is due to adjacent sites having very close on-site potential and thus having an electron resonate between the two sites.

adjacent to the central bond, $\left|w_{i}-w_{j}\right|$. The data clearly shows that configurations with $S_{c} \approx \log 2$ occur predominantly when the difference $\left|w_{i}-w_{j}\right|$ is very small. We conclude from this correlation that the peaks at $S_{c} \approx \log 2$ are an artifact due to configurations that favor electrons at the center to spread over two sites adjacent to the central cut.

\section{Appendix C: Localization in Fock space}

In this section, we will discuss some numerical results on the Fock space localization problem introduced in Section II. For sake of readability, we repeat the derivation of Eqn. (4) here, giving some additional details. We begin by solving the non-interacting part $H_{0}$ of Hamiltonian (2) to obtain the eigenvalues $\varepsilon_{n}$ and eigenvectors $\phi_{n}(i)$. We can then rewrite

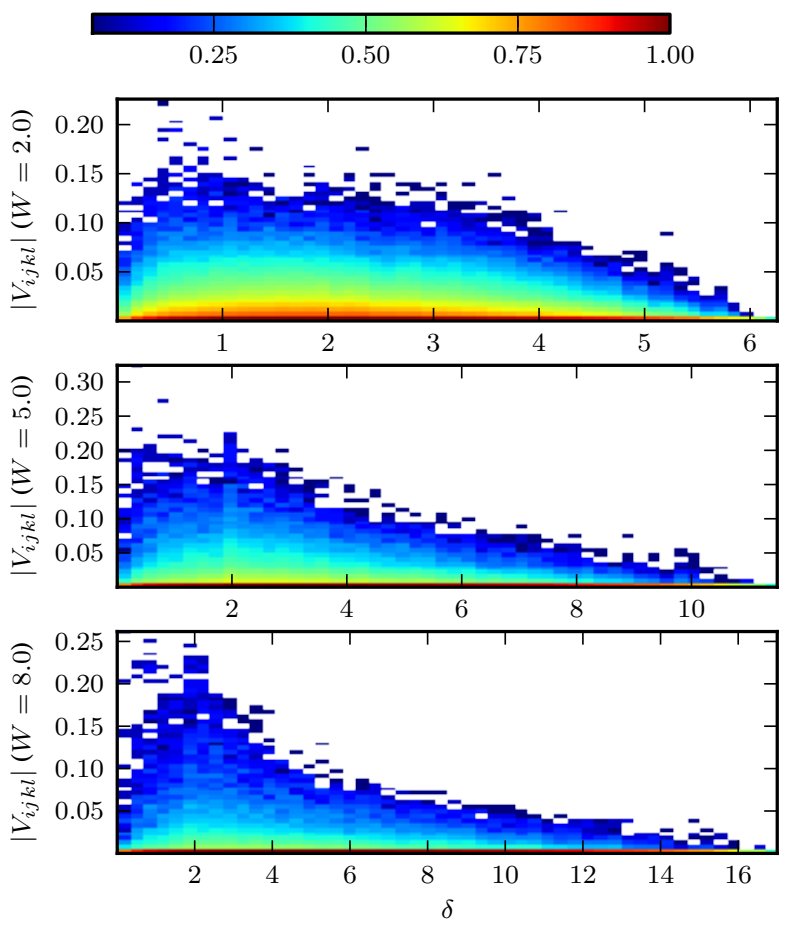

FIG. 8. Normalized logarithm of the histogram, $\ln H$, of the strength of four-fermion terms $\left|V_{i j k l}\right|$ vs. the energy difference $\delta$ of the single-particle orbitals connected by this interaction term, cf. (C4). Results are for $L=24$ and $t=V=1$ and accumulated over 100 disorder realizations. White regions correspond to $H=0$.
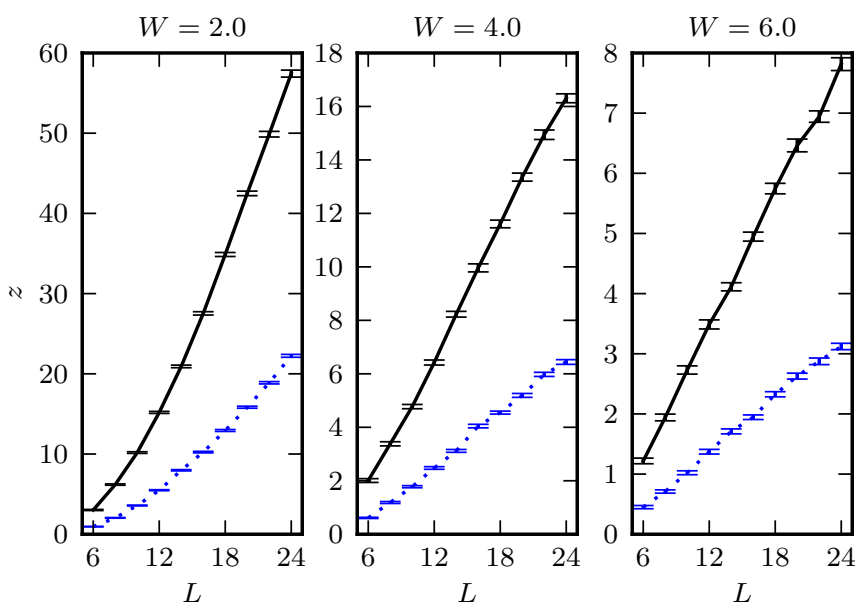

FIG. 9. Scaling of the effective coordination number $z$ of Eqn. (5) with system size $L$ at half filling $N=L / 2$ for $V=1.2$ (black, solid lines) and $V=0.4$ (blue, dotted lines).

the problem as follows (cf. (3)):

$$
H=\sum \varepsilon_{n} d_{n}^{\dagger} d_{n}+\sum_{i j k l} V_{i j k l} d_{i}^{\dagger} d_{j}^{\dagger} d_{k} d_{l},
$$




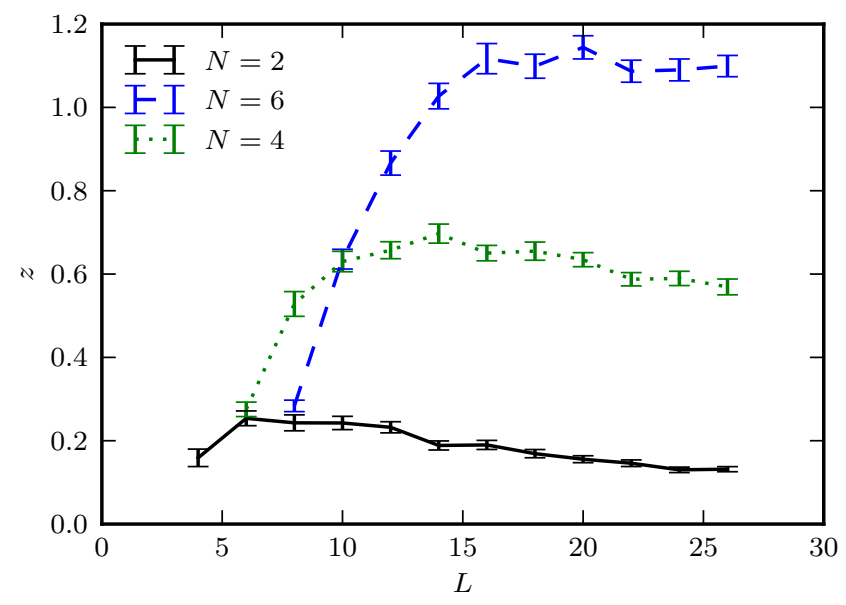

FIG. 10. Scaling of the effective coordination number $z$ of Eqn. (5) with system size $L$ at particle number $N$, for $N=2,4,6$. Calculations are performed at $W=8$ and $V=0.4$, i.e. in a strongly localized regime.

where $d_{n}^{\dagger}$ creates a fermion in the single-particle state $\phi_{n}(i)$, and the coefficients of the four-fermion term are

$$
V_{i j k l}=V \sum_{p=1}^{L-1} \phi_{i}^{*}(p) \phi_{j}^{*}(p+1) \phi_{k}(p+1) \phi_{l}(p) .
$$

This defines a Fock space model, in which we interpret the Slater determinants formed by filling single-particle eigenstates as sites of our new model. We denote such a Slater determinant as $|\vec{\alpha}\rangle=\prod\left(d_{n}^{\dagger}\right)^{\alpha_{n}}|0\rangle$, where $\vec{\alpha}$ is an occupationnumber vector and $d_{n}^{\dagger}=\sum_{i} \phi_{n}(i) c_{i}^{\dagger}$. There are $2^{L}$ such sites in the full Fock space; restricting to half filling, we are left with $\left(\begin{array}{c}L \\ L / 2\end{array}\right)$ sites. We thus obtain the Fock space model (cf. (4))

$$
\begin{aligned}
H & =\sum_{\vec{\alpha}} \mu_{\alpha}|\vec{\alpha}\rangle\left\langle\vec{\alpha}\left|+\sum_{\vec{\alpha} \neq \vec{\beta}} V_{\beta \alpha}\right| \vec{\beta}\right\rangle\langle\vec{\alpha}| \\
\mu_{\alpha} & =\sum_{n} \alpha_{n} \varepsilon_{n}+V_{\alpha \alpha} \\
V_{\beta \alpha} & =\sum_{i j k l} V_{i j k l}\left\langle\vec{\beta}\left|d_{i}^{\dagger} d_{j}^{\dagger} d_{k} d_{l}\right| \vec{\alpha}\right\rangle .
\end{aligned}
$$

We will now numerically analyze the structure of the effective hopping terms $V_{\alpha \beta}$. Since we are assuming that we only have two-body interactions, the many-body states $|\alpha\rangle$ and $|\beta\rangle$ can only differ in the occupations of four of the single particle states $\phi_{i}, \phi_{j}, \phi_{k}, \phi_{l}$, which directly relates the $V_{\alpha \beta}$ to the $V_{i j k l}$. As discussed previously, due to the localization of single-particle states $\phi_{n}(i)$, the matrix elements $V_{i j k l}$ fall off exponentially with separation between the states. We now want to confirm numerically that they also fall off exponentially with the difference in single-particle energies.

To this end, let us consider only terms where $i \neq j \neq k \neq l$ and define

$$
\begin{array}{r}
\delta=\min \left\{\max \left(\left|\varepsilon_{i}-\varepsilon_{k}\right|,\left|\varepsilon_{j}-\varepsilon_{l}\right|\right),\right. \\
\left.\max \left(\left|\varepsilon_{i}-\varepsilon_{l}\right|,\left|\varepsilon_{j}-\varepsilon_{k}\right|\right)\right\} .
\end{array}
$$

This defines a natural measure for the energy difference of the single-particle eigenstates involved in the term $V_{i j k l}$. In Fig. 8, we show a histogram of how the strength of $\left|V_{i j k l}\right|$ depends on this distance $\delta$. Following Basko et al. ${ }^{1,2}$, we expect that terms with large $\delta$ are strongly suppressed in a sufficiently strongly disordered system. This is nicely confirmed in our data: for large enough $\delta$, only very weak terms $\left|V_{i j k l}\right| \ll 1$ occur, whereas for small $\delta$ terms of all strength up to $\left|V_{i j k l}\right| \sim$ $\mathcal{O}(1)$ occur. Note that for any choice of disorder strength, most of the terms are very small. For terms of the form $V_{i k k j}$, i.e. where only one fermions hops, very similar results are obtained with $\delta=\left|\varepsilon_{i}-\varepsilon_{j}\right|$; the probability of having terms of strength $\mathcal{O}(1)$ in this case is independent of $\left|\varepsilon_{i}-\varepsilon_{k}\right|$ and $\left|\varepsilon_{j}-\varepsilon_{k}\right|$. Terms of the form $V_{i j i j}$, which are diagonal, have a strength that is largely independent of $\left|\varepsilon_{i}-\varepsilon_{j}\right|$.

Turning our attention to the structure of the hopping problem of (C3), we now examine the effective coordination number previously defined in (5):

$$
z=\left\langle z_{\alpha}\right\rangle, \quad z_{\alpha}=\sum_{\beta \neq \alpha} \frac{V_{\beta \alpha}}{\left|\mu_{\beta}-\mu_{\alpha}\right|}
$$

Due to practical considerations, we only evaluate terms with $\left|V_{i j k l}\right|>\epsilon$ with $\epsilon=10^{-3}$; our estimate for $z$ can thus be considered a lower bound, but we have confirmed that the estimate is independent of $\epsilon$ within statistical error bars. Our results for a half-filled system are shown in Fig. 9, which shows the scaling approaches $z \sim L$ largely independently of the parameters of the model. To obtain a better understanding, we study the dependence of $z$ on $L$ at fixed particle number instead of fixed filling fraction. We show $z$ as a function of $L$ in Fig. 10 for system sizes up to $L=26$ and fixed particle number $N=2,4,6$. In all three cases, a saturation of $z$ is observed for $L=c N$, where $c$ is a constant which is roughly $c \approx 3$ for our choice of parameters. This coefficient will depend on the single-particle localization length and therefore on the disorder strength $W$.
1 D. M. Basko, I. L. Aleiner, and B. L. Altshuler, Annals of Physics 321, 1126 (2006).

2 D. M. Basko, I. L. Aleiner, and B. L. Altshuler, "On the problem of many-body localization," arXiv:cond-mat/0602510.

3 P. W. Anderson, Phys. Rev. 109, 1492 (1958).
${ }^{4}$ D. M. Basko, I. L. Aleiner, and B. L. Altshuler, Phys. Rev. B 76, 052203 (2007).

5 J. M. Deutsch, Phys. Rev. A 43, 2046 (1991).

6 M. Srednicki, Phys. Rev. E 50, 888 (1994).

7 B. L. Altshuler, Y. Gefen, A. Kamenev, and L. S. Levitov, Phys. 
Rev. Lett. 78, 2803 (1997).

8 R. Berkovits and Y. Avishai, Phys. Rev. Lett. 80, 568 (1998).

${ }^{9}$ C. Mejía-Monasterio, J. Richert, T. Rupp, and H. A. Weidenmüller, Phys. Rev. Lett. 81, 5189 (1998).

10 V. Oganesyan and D. A. Huse, Phys. Rev. B 75, 155111 (2007).

11 A. Pal and D. A. Huse, Phys. Rev. B 82, 174411 (2010).

12 S. Iyer, V. Oganesyan, G. Refael, and D. A. Huse, Preprint (2012), arXiv:1212.4159.

13 M. Z̆nidarič, T. Prosen, and P. Prelovšek, Phys. Rev. B 77, 064426 (2008).

${ }^{14}$ G. D. Chiara, S. Montangero, P. Calabrese, and R. Fazio, J. Stat. Mech. , P03001 (2006).

15 J. H. Bardarson, F. Pollmann, and J. E. Moore, Phys. Rev. Lett. 109, 017202 (2012).

${ }^{16}$ R. Vosk and E. Altman, Phys. Rev. Lett. 110, 067204 (2013).

17 M. Serbyn, Z. Papić, and D. Abanin, Preprint (to appear in Phys. Rev. Lett.) (2013), arXiv:1304.4605.

18 D. A. Huse and V. Oganesyan, Preprint (2013), arXiv:1305.4915.

${ }^{19}$ C. K. Burrell and T. J. Osborne, Phys. Rev. Lett. 99, 167201 (2007).

20 J. Fröhlich and T. Spencer, Comm. Math. Phys. 88, 151 (1983).

21 J. Eisert, M. Cramer, and M. B. Plenio, Rev. Mod. Phys. 82, 277 (2010).

22 R. Berkovits, Phys. Rev. Lett. 108, 176803 (2012).

${ }^{23}$ M. M. Wolf, F. Verstraete, M. B. Hastings, and J. I. Cirac, Phys. Rev. Lett. 100, 070502 (2008).

${ }^{24}$ G. Vidal, J. I. Latorre, E. Rico, and A. Kitaev, Phys. Rev. Lett. 90, 227902 (2003).

25 S. Chakravarty, Int. J. Mod. Phys. B 24, 1823 (2010).

${ }^{26}$ D. A. Huse, R. Nandkishore, V. Oganesyan, A. Pal, and S. L. Sondhi, Preprint (2013), arXiv:1304.1158.

27 C. Monthus and T. Garel, Phys. Rev. B 81, 134202 (2010).

${ }^{28}$ I. Aleiner, P. Brouwer, and L. Glazman, Physics Reports 358, 309 (2002).

29 A. D. Mirlin, Physics Reports 326, 259 (2000).

${ }^{30}$ C. Helstrom, "Quantum detection and estimation theory," (Academic, 1976).

31 C. Fuchs and J. van de Graaf, IEEE Trans. Inf. Theory 45, 1216 (1999).

${ }^{32}$ B. Bauer, M. Troyer, V. W. Scarola, and K. B. Whaley, Phys. Rev. B 81, 085118 (2010).

33 F. Verstraete and J. I. Cirac, Phys. Rev. B 73, 094423 (2006).

${ }^{34}$ M. B. Hastings, Phys. Rev. B 76, 035114 (2007).

35 M. B. Hastings, J. Stat. Mech., P08024 (2007).

${ }^{36}$ M. Serbyn, Z. Papić, and D. Abanin, Preprint (2013), arXiv:1305.5554.

37 Z.-C. Gu and X.-G. Wen, Phys. Rev. B 80, 155131 (2009).

${ }^{38}$ Note that for an SPT phase, such a decomposition is possible if one first applies a finite-depth unitary transformation that breaks the symmetry.

39 M. Rigol and M. Srednicki, Phys. Rev. Lett. 108, 110601 (2012).

40 J. D. Bekenstein, Phys. Rev. D 7, 2333 (1973).

${ }^{41}$ L. Bombelli, R. K. Koul, J. Lee, and R. D. Sorkin, Phys. Rev. D 34, 373 (1986).

42 M. Srednicki, Phys. Rev. Lett. 71, 666 (1993).

43 C. Holzhey, F. Larsen, and F. Wilczek, Nucl. Phys. B 424, 443 (1994).

44 P. Calabrese and J. Cardy, J. Stat. Mech., P06002 (2004).

45 G. Refael and J. E. Moore, Phys. Rev. Lett. 93, 260602 (2004).

${ }^{46}$ M. B. Plenio, J. Eisert, J. Dreißig, and M. Cramer, Phys. Rev. Lett. 94, 060503 (2005).

47 M. M. Wolf, Phys. Rev. Lett. 96, 010404 (2006).

48 D. Gioev and I. Klich, Phys. Rev. Lett. 96, 100503 (2006).

${ }^{49}$ M. B. Hastings, Phys. Rev. Lett. 107, 210501 (2011).

${ }^{50}$ M. B. Hastings and X.-G. Wen, Phys. Rev. B 72, 045141 (2005).

51 C. Nayak, S. H. Simon, A. Stern, M. Freedman, and S. D. Sarma, Rev. Mod. Phys. 80, 1083 (2008).

52 A. Kitaev, Annals of Physics 321, 2 (2006).

53 S. Trebst, P. Werner, M. Troyer, K. Shtengel, and C. Nayak, Phys. Rev. Lett. 98, 070602 (2007).

54 A. Hamma and D. A. Lidar, Phys. Rev. Lett. 100, 030502 (2008).

55 J. Vidal, S. Dusuel, and K. P. Schmidt, Phys. Rev. B 79, 033109 (2009).

56 J. Vidal, R. Thomale, K. P. Schmidt, and S. Dusuel, Phys. Rev. B 80, 081104 (2009).

57 I. S. Tupitsyn, A. Kitaev, N. V. Prokof'ev, and P. C. E. Stamp, Phys. Rev. B 82, 085114 (2010).

58 S. Dusuel, M. Kamfor, R. Orús, K. P. Schmidt, and J. Vidal, Phys. Rev. Lett. 106, 107203 (2011).

59 C. Stark, L. Pollet, A. Imamoğlu, and R. Renner, Phys. Rev. Lett. 107, 030504 (2011).

${ }^{60}$ B. Bauer and C. Nayak, (2013).

61 J. R. Wootton and J. K. Pachos, Phys. Rev. Lett. 107, 030503 (2011).

${ }^{62}$ S. Bravyi and R. König, Comm. Math. Phys. 316, 641 (2012).

${ }^{63}$ E. Dennis, A. Kitaev, A. Landahl, and J. Preskill, J. Math. Phys. 43, 4452 (2002).

${ }^{64}$ R. Alicki, M. Horodecki, P. Horodecki, and R. Horodecki, Open Syst. Inf. Dyn. 17, 1 (2010).

${ }^{65}$ R. Alicki, M. Fannes, and M. Horodecki, J. Phys. A: Math. Theor. 42, 065303 (2008).

${ }^{66}$ R. Alicki and M. Horodecki, Preprint (2006), arXiv:quant$\mathrm{ph} / 0603260$.

${ }^{67}$ S. Bravyi, M. B. Hastings, and F. Verstraete, Phys. Rev. Lett. 97, 050401 (2006).

${ }^{68}$ M. Levin and X.-G. Wen, Phys. Rev. Lett. 96, 110405 (2006).

${ }^{69}$ B. Bauer et al., J. Stat. Mech., P05001 (2011). 